\title{
Vertical Velocity Profiles in Convectively Coupled Equatorial Waves and MJO: New Diagnoses of Vertical Velocity Profiles in the Wavenumber-Frequency Domain
}

\author{
KUNIAKI INOUE \\ NASA Goddard Institute for Space Studies, New York, New York, and Universities Space Research Association, Columbia, Maryland, \\ and Lamont-Doherty Earth Observatory, Columbia University, Palisades, New York \\ ÁNGEL F. ADAMES \\ Department of Climate and Space Science and Engineering, University of Michigan, Ann Arbor, Michigan \\ KAZUAKI YASUNAGA \\ Department of Earth Science, Graduate School of Science and Engineering, University of Toyama, Toyama, \\ and Japan Agency for Marine-Earth Science and Technology, Yokosuka, Japan
}

(Manuscript received 31 July 2019, in final form 23 March 2020)

\begin{abstract}
A new diagnostic framework is developed and applied to ERA-Interim to quantitatively assess vertical velocity (omega) profiles in the wavenumber-frequency domain. Two quantities are defined with the first two EOF-PC pairs of omega profiles in the tropical ocean: a top-heaviness ratio and a tilt ratio. The top-heaviness and tilt ratios are defined, respectively, as the cospectrum and quadrature spectrum of PC1 and PC2 divided by the power spectrum of PC1. They represent how top-heavy an omega profile is at the convective maximum, and how much tilt omega profiles contain in the spatiotemporal evolution of a wave. The top-heaviness ratio reveals that omega profiles become more top-heavy as the time scale (spatial scale) becomes longer (larger). The MJO has the most top-heavy profile while the eastward inertio-gravity (EIG) and westward inertiogravity (WIG) waves have the most bottom-heavy profiles. The tilt ratio reveals that the Kelvin, WIG, EIG, and mixed Rossby-gravity (MRG) waves, categorized as convectively coupled gravity waves, have significant tilt in the omega profiles, while the equatorial Rossby (ER) wave and MJO, categorized as slow-moving moisture modes, have less tilt. The gross moist stability (GMS), cloud-radiation feedback, and effective GMS were also computed for each wave. The MJO with the most top-heavy omega profile exhibits high GMS, but has negative effective GMS due to strong cloud-radiation feedbacks. Similarly, the ER wave also exhibits negative effective GMS with a top-heavy omega profile. These results may indicate that top-heavy omega profiles build up more moist static energy via strong cloud-radiation feedbacks, and as a result, are more preferable for the moisture mode instability.
\end{abstract}

\section{Introduction}

A significant fraction of tropical clouds are organized in aggregated systems over a wide range of spatial and temporal scales. On daily to intraseasonal time scales, those aggregated systems are often organized by tropical wave disturbances that include the convectively coupled equatorial waves (CCEWs; Takayabu 1994; Wheeler and Kiladis 1999; Kiladis et al. 2009) and the MaddenJulian oscillation (MJO; Madden and Julian 1971, 1972). The CCEWs consist of different members: Kelvin, equatorial Rossby (ER), westward inertio-gravity (WIG),

Corresponding author: Kuniaki Inoue, kuni.inoue22@gmail.com eastward inertio-gravity (EIG), and mixed Rossbygravity (MRG) waves. They are linearly independent modes of the shallow-water equations in the equatorial beta plane (Matsuno 1966). In addition to the aforementioned modes, a tropical depression (TD)-type wave, which is not a solution of the shallow-water equations, also shares many similarities to the CCEWs.

The CCEWs, TD-type wave, and MJO are linked to high-impact weather events including tropical cyclones and extreme rainfall and flooding. They also impact climate phenomena such as the global monsoon systems and ENSO-refer to review papers by Kiladis et al. (2009) for the CCEWs and TD-type wave and by Zhang (2013) for the MJO. 
TABLE 1. Categorization of the CCEWs, TD-type wave, and MJO suggested by Raymond et al. (2015).

\begin{tabular}{llll}
\hline \hline \multicolumn{1}{c}{ Category } & \multicolumn{1}{c}{ Mechanism } & Vertical structure & \multicolumn{1}{c}{ Members } \\
\hline Fast-moving mode & Convectively coupled gravity wave & Tilted & Kelvin, WIG, EIG, MRG \\
Slow-moving mode & Moisture mode & Vertically stacked & MJO, ER, TD type \\
\hline
\end{tabular}

Despite their importance, the representation of these tropical disturbances in general circulation models (GCMs) has remained challenging (e.g., Lin et al. 2006; Straub et al. 2010; Kim et al. 2009; Benedict et al. 2013; Hung et al. 2013; Jiang et al. 2015) even though there has been significant progress (e.g., Hung et al. 2013). More specifically, the difficulty may exist in simulating all the members of the CCEWs and the MJO in one GCM. For instance, one model can simulate a certain member of the CCEWs, but is unable to simulate the MJO, or vice versa [e.g., Figs. 4 and 5 in Hung et al. (2013)]. This fact indicates that each member of the CCEWs and the MJO may be regulated by different mechanisms, and a model parameterization favorable for one type of wave may be unfavorable for the other types of waves.

Existing literature suggests that the tropical waves can be categorized into two groups. Based on the horizontal structures of the dynamical fields, Kiladis et al. (2009) and Yasunaga and Mapes (2012a,b) suggested that the members of the CCEWs and the TD-type wave can be categorized into 1) divergent-type and 2) rotational-type waves. According to this categorization, the Kelvin, WIG, and EIG waves belong to the divergent type while the ER, MRG, and TD-type waves belong to the rotational type.

On the other hand, based on more theoretical arguments, Raymond et al. (2015) and Herman et al. (2016) categorized the CCEWs, TD-type wave, and MJO into 1) fast-moving and 2) slow-moving modes. Following their categorization, the Kelvin, WIG, EIG, and MRG waves are categorized as the fast-moving modes while the ER, TD-type waves, and MJO are grouped as the slow-moving modes.

Theoretically, the fast-moving modes correspond to the convectively coupled (CC) gravity waves and the slow-moving modes correspond to the moisture modes (Raymond and Fuchs 2007), as summarized in Table 1. Each of these distinct modes has been extensively studied by the past theoretical literature. For instance, the CC gravity wave modes have been studied for elucidating the mechanisms of the Kelvin and WIG waves (e.g., Mapes 2000; Khouider and Majda 2006; Raymond and Fuchs 2007; Kuang 2008b). Theories of the moisture modes have been proposed by many studies aiming to elucidate the basic mechanisms of the MJO (e.g., Sobel and Gildor 2003; Fuchs and Raymond 2007; Raymond and Fuchs 2007; Sobel and Maloney 2013; Sugiyama
2009; Adames and Kim 2016; Fuchs and Raymond 2017). Some variations of the moisture modes have been proposed for elucidating the mechanisms of the monsoon depression (Adames and Ming 2018) and the ER wave (Fuchs-Stone et al. 2019). Raymond and Fuchs (2007) and its variational study (Fuchs et al. 2012) claimed that the CC gravity wave mode and the moisture mode are governed by fundamentally different mechanisms.

In all of the theoretical literature listed above, one of the most important ingredients for the theory is the vertical velocity (omega) profile, which is closely related to the latent heating profile. More specifically, two properties of omega profiles are crucial: 1) tilted structures with height, and 2) the top-heaviness or bottomheaviness of omega profiles.

Raymond and Fuchs (2007) showed that one of the most prominent differences between the CC gravity wave mode (or the fast-moving mode) and the moisture mode (or the slow-moving mode) is the amount of the tilt of omega profiles in their spatiotemporal evolution; the CC gravity wave mode has significant tilts whereas the moisture mode has vertically stacked profiles. The other theoretical literature for the CC gravity wave modes (Mapes 2000; Khouider and Majda 2006; Kuang 2008b) also suggested that the tilted structure is a key to the instability and propagation of the CC gravity waves.

The idea of the importance of the tilt was augmented by some studies with cloud-system resolving models (e.g., Peters and Bretherton 2006; Kuang 2008a). Many observational studies also documented tilted structures in the CCEW members that belong to the fast-moving modes: Kelvin waves (e.g., Straub and Kiladis 2002, 2003; Kiladis et al. 2009), WIG waves (e.g., Takayabu et al. 1996; Haertel and Kiladis 2004; Haertel et al. 2008; Kiladis et al. 2009; Inoue and Back 2015a; Sumi and Masunaga 2016, 2019), EIG waves (e.g., Kiladis et al. 2009), and MRG waves (e.g., Liebmann and Hendon 1990; Takayabu and Nitta 1993; Kiladis et al. 2009). In contrast, Kiladis et al. (2009) showed vertically stacked structures of the ER wave, which belongs to the slowmoving modes.

Some studies pointed out tilted omega (or latent heating) structures in the MJO, which belongs to the slow-moving moisture modes (e.g., Lin et al. 2004; Kiladis et al. 2005; Haertel et al. 2008; Adames and Wallace 2014). Mapes et al. (2006) claimed the "self-similarity" 
of the tilted structures among the CCEWs (excluding the ER wave) and the MJO. However, Inoue and Back (2015a) compared the tilts of the omega profiles in the WIG wave and the MJO using field campaign data, and found that the MJO is much more vertically stacked than the WIG wave, speculating that omega profiles get more vertically stacked as the time scale gets longer. This speculation is tested in this study in a more rigorous way.

The instability of the slow-moving moisture modes is set by a quantity called the gross moist stability (GMS). The GMS represents the efficiency of the export of moist static energy (MSE) via large-scale circulations associated with moist convection (e.g., Neelin and Held 1987; Raymond et al. 2009). High values of the GMS are associated with a convective system that efficiently exports MSE, and are believed to be less favorable for the growth of the moisture modes. The value of the GMS is primarily determined by the shape of omega profiles (e.g., Sobel 2007; Raymond et al. 2009; Inoue and Back 2015a,b). In general, a top-heavy profile is associated with a high value of the GMS whereas a bottom-heavy profile is associated with a low value. Therefore, according to the moisture mode theories, a bottom-heavy omega profile with low GMS may be more favorable for the growth of the moisture modes.

Based on this idea, Kuang (2011) (K11, hereafter) proposed a hypothesis about the instability of the MJO and its scale selectivity. With a limited-domain cloudsystem-resolving model that is coupled with parameterized large-scale circulations in the steady state, K11 found that as the spatial scale becomes larger, the GMS becomes lower via more bottom-heavy omega profiles. And K11 hypothesized that the MJO, which has a large spatial scale, may be more preferably excited via lower values of the GMS. However, Sakaeda and Roundy (2016), who analyzed the GMS on various temporal and spatial scales, found no such relationship between the value of the GMS and spatial scales. We examine K11's hypothesis in this study.

As discussed above, the tilt and top-heaviness of omega profiles may be crucial for the mechanisms of the CCEWs and MJO. Therefore, we need to investigate those two properties among all the waves in a consistent framework, and answer specific questions as follows:

1) Which waves have a more top-heavy or bottomheavy omega profile shape?

2) Which wave has tilted (or vertically stacked) omega profiles in its spatiotemporal evolution?

3) Is there any distinction in the amount of the tilt of omega profiles between the fast-moving modes and the slow-moving modes?
4) Is there any time-scale dependency in the amount of the tilt of omega profiles?

For answering these questions, we propose a new diagnostic framework in which we can quantitatively assess the tilt and the top-heaviness of omega profiles among the different waves.

The remainder of this study is structured as follows. In section 2, we describe the data used and the procedures of the space-time Fourier transformation. In section 3, the quantities called the "top-heaviness ratio" and the "tilt ratio" are defined, and we demonstrate how those quantities represent the structures of omega profiles in the spatiotemporal evolution of a wave. In section 4 , the top-heaviness and tilt ratios are computed for each wave, answering the four questions posed above. Furthermore, in this section, the instability of the moisture modes is investigated more. In section 5, we discuss the caveats of this study, and also physical interpretations of different structures of omega profiles in each wave. Finally, section 6 summarizes this study.

\section{Data and methods}

\section{a. Data}

We used the data of vertical pressure velocity (omega), temperature, geopotential height, specific humidity, and surface pressure from the European Centre for Medium-Range Weather Forecasts (ECMWF) interim reanalysis (ERA-Interim, hereafter ERA-I; Dee et al. 2011). First, the raw data with 6-hourly and $\sim 0.7^{\circ} \times 0.7^{\circ}$ resolution was regridded onto a daily and $\sim 1.4^{\circ} \times 1.4^{\circ}$ resolution with temporal and spatial averages. Second, because ERA-I's three-dimensional data contain artificial values below the surface pressure that are the product of the extrapolation from a model to pressure coordinate, we replaced those artificial values at each time and location with zero. Finally, we truncated the data above $100 \mathrm{hPa}$, retaining the values at 27 vertical levels from 1000 to $100 \mathrm{hPa}$.

In addition to the analysis data, we also utilized the data of top thermal radiation [aka outgoing longwave radiation (OLR)], top solar radiation, surface thermal radiation, and surface solar radiation from the $12-\mathrm{h}$ accumulated forecast data in the ERA-I dataset. Daily averages were calculated by adding two consecutive 12-h accumulated forecast values in the same day and dividing it by $24 \mathrm{~h}$. Those daily average forecast data were regridded onto the same spatial grid as the other variables. 
As supplemental data, we also used the daily NOAA Interpolated OLR data ${ }^{1}$ with a $2.5^{\circ} \times 2.5^{\circ}$ grid (Liebmann and Smith 1996), which were compared with the OLR data from ERA-I.

For avoiding the topographic effects on the analyses, especially for the analyses of the omega profiles, we masked out all the values over land. We investigated the 33-yr-long period ranging from 1984 to 2016 of each variable over the tropical belt $\left(15^{\circ} \mathrm{N}-15^{\circ} \mathrm{S}\right)$.

\section{b. Space-time Fourier transformation}

This study is primarily based on the discrete spacetime Fourier transformation. We can express the longitude $x$ in the unit of radians and time $t$ discretely as $x_{m}=$ $m \Delta x(m=1,2, \ldots, M)$ and $t_{n}=n \Delta t(n=1,2, \ldots, N)$, where $\Delta x$ and $\Delta t$ are the zonal and temporal increment lengths of the gridded data. Then, for each latitude and time series segment, we can formally write the discrete space-time Fourier transformation of an arbitrary variable $a\left(x_{m}, t_{n}\right)$ as

$$
\begin{aligned}
\mathscr{F}\left\{a\left(x_{m}, t_{n}\right)\right\} \equiv & A\left(k, \sigma_{l}\right) \\
= & \frac{1}{M N} \sum_{m=1}^{M} \sum_{n=1}^{N} a\left(x_{m}, t_{n}\right) \\
& \times \exp \left\{-i\left(k x_{m}-\sigma_{l} t_{n}\right)\right\} .
\end{aligned}
$$

Here $A\left(k, \sigma_{l}\right)$ is the Fourier coefficient of $a\left(x_{m}, t_{n}\right)$, as a function of a zonal wavenumber $k$ and a temporal angular frequency $\sigma_{l}$ that is defined as

$$
\sigma_{l} \equiv \frac{2 \pi l}{N \Delta t}
$$

Since $M \Delta x=2 \pi$, we can rearrange Eq. (1) as

$$
A(k, l)=\frac{1}{M N} \sum_{m=1}^{M} \sum_{n=1}^{N} a\left(x_{m}, t_{n}\right) \exp \left\{-i\left(\frac{2 \pi k m}{M}-\frac{2 \pi l n}{N}\right)\right\} .
$$

This form is more consistent with a programing code of the Fourier transformation. The temporal wavenumber $l$ can be converted into the temporal frequency of units of cycles per day by dividing it by $N \Delta t$.

The space-time Fourier transformation for each latitude and time series segment was computed with the procedures commonly used in the tropical meteorology (e.g., Wheeler and Kiladis 1999; Hendon and Wheeler 2008). 1) From the entire time series, we removed the

\footnotetext{
${ }^{1}$ The data used are provided by the NOAA/OAR/ESRL PSD, Boulder, Colorado, from their website at https://www.esrl.noaa.gov/ psd/.
}

temporal mean and the three leading harmonics of the annual cycle [i.e., the harmonics with the frequencies of 1,2 , and 3 (365 days $)^{-1}$ ] computed with the least squares method. 2) The time series were separated into the antisymmetric and symmetric components around the equator as $a_{a}(x, y, t)=[a(x, y, t)-a(x,-y, t)] / 2$ and $a_{s}(x, y, t)=[a(x, y, t)+a(x,-y, t)] / 2$ for $y \neq 0$ (not on the equator), where $a_{a}$ and $a_{s}$ are the antisymmetric and symmetric component, respectively. 3) We subset the time series into segments with the segment length of 256 days and the overlap length of 77 days; as a result, we obtained 66 segments of time series. 4) From each segment, a linear trend computed with the least squares method was removed. 5) The edges of the time series segments were tapered with the cosine window (aka the Hann window) with the tapering length of 12 days from each side. 6) Finally, we computed the space-time Fourier transformation with Eq. (3) for each latitude and time series segment.

For masking out the land from the spectral analyses, we first replaced the values over land in the original time series with a nonnumber. After separating into the antisymmetric and symmetric components, we replaced the nonnumbers with zero, a process called zero padding. The Fourier transformation procedure followed thereafter.

\section{c. Calculation of regional Fourier transformation}

In section $4 \mathrm{e}$, we also conducted a regional spectral analysis for examining the geographic dependency of our results. A regional Fourier transformation localized by a zonal sector was calculated with the zonal tapering method from Dias et al. (2013) where the Hann window is applied not only to the temporal dimension, but also to the zonal dimension in step 5 above. Since one of our primary interests is the $\mathrm{MJO}$, we chose the zonal sector of the warm pool region ranging from $60^{\circ} \mathrm{E}$ to $180^{\circ}$. The values outside of this sector were replaced with zero, and the edges of the sector were tapered with the Hann window with the tapering length of $\sim 10^{\circ}$ from each side, followed by the calculation of the space-time Fourier transformation.

\section{d. Calculation of a fractional quantity in the wavenumber-frequency domain}

In this study, we primarily investigated fractional quantities as a function of zonal wavenumbers and temporal frequencies. These are written in the form of

$$
\frac{A(k, l)}{B(k, l)},
$$

where $A$ and $B$ are arbitrary complex quantities. When computing such a fractional quantity as a 
function of $k$ and $l$, we consistently followed the procedures below:

1) $A$ and $B$ were computed separately for each latitude and time series segment;

2) $A$ and $B$ were separately averaged over the latitudes and the time series segments;

3 ) the averaged $A$ and $B$ were separately smoothed with 3 passes of a 1-2-1 running-mean filter in the temporal-frequency dimension (along a constant zonal wavenumber) for improving the display and increasing the degrees of freedom; and

4) finally, we took the ratio between the averagedsmoothed $A$ and $B$.

Similar procedures have been employed, for instance, for computing the coherence between two quantities (e.g., Wheeler and Kiladis 1999; Hendon and Wheeler 2008; Yasunaga and Mapes 2012a; Yasunaga et al. 2019).

\section{Top-heaviness and tilt ratios}

\section{a. Definition}

In this study, we propose a new diagnostic method to assess the structures of the spatiotemporal evolution of omega profiles in tropical wave disturbances. More specifically, we define two quantities, which we refer to as the "top-heaviness" ratio and the "tilt ratio," as a function of zonal wavenumbers and temporal frequencies.

Following the notation of Back and Bretherton (2009), we approximate the omega profiles with the two leading profiles:

$$
\omega(x, y, p, t) \simeq o_{1}(x, y, t) \Omega_{1}(p)+o_{2}(x, y, t) \Omega_{2}(p),
$$

where $\Omega_{1}$ and $\Omega_{2}$ are the first and second empirical orthogonal functions (EOFs) illustrated in Fig. 1-hereafter, EOF1 and EOF2, respectively; and $o_{1}$ and $o_{2}$ represent the corresponding principle components (PCs) - hereafter, $\mathrm{PC} 1$ and PC2, respectively. In ERA-I, these two EOF-PC pairs explain $83.5 \%$ of the total spatiotemporal variance of the omega profiles in the tropical oceanic belt (from $15^{\circ} \mathrm{S}$ to $15^{\circ} \mathrm{N}$ excluding the land); the first and second pairs explain $67.1 \%$ and $16.4 \%$ of the variance, respectively.

We take the Fourier transformation of the $j$ th PC, $o_{j}(j=1,2, \ldots, 27)$, and write them as

$$
\mathscr{T}\left\{o_{j}\left(x_{m}, t_{n}\right)\right\} \equiv O_{j}(k, l) .
$$

By using them, we define a complex parameter $\tau$ as a ratio between the Fourier coefficients of $\mathrm{PC} 2$ and $\mathrm{PC} 1$, or mathematically,

$$
O_{2}(k, l) \equiv \tau O_{1}(k, l),
$$

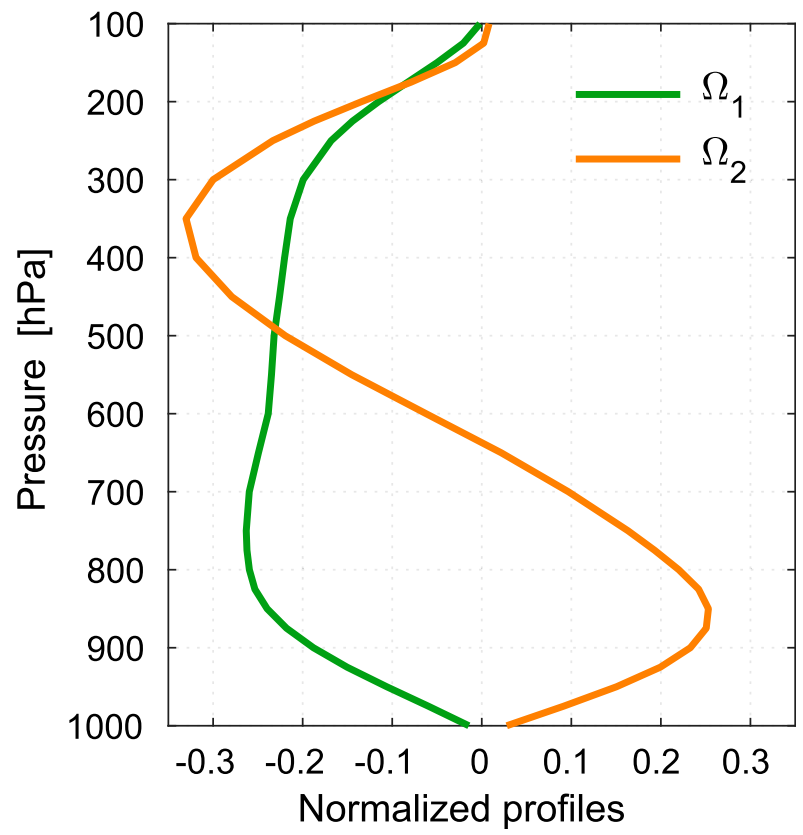

FIG. 1. EOF1 $\left(\Omega_{1}\right)$ and EOF2 $\left(\Omega_{2}\right)$ of the omega profiles in ERA-I over the tropical oceanic belt. The amplitudes of the EOFs were normalized into a unit vector.

or

$$
\tau \equiv \frac{O_{2} O_{1}^{*}}{O_{1} O_{1}^{*}},
$$

where the star mark represents the complex conjugate. Since $\tau$ is a complex number, it has two components:

$$
\tau_{r} \equiv \operatorname{Re}(\tau) ; \quad \tau_{i} \equiv \operatorname{Im}(\tau),
$$

where $\mathrm{Re}$ and $\mathrm{Im}$ are real and imaginary components of a complex number, respectively. The parameters $\tau_{r}$ and $\tau_{i}$ are the cospectrum and quadrature spectrum of PC1 and PC2, respectively, divided by the power spectrum of PC1.

As demonstrated in the next, $\tau_{r}$ represents how topheavy (or bottom-heavy) an omega profile is at the convective maximum when the amplitude of $\mathrm{PC} 1$, which is a good proxy of precipitation, is the greatest; and $\tau_{i}$ represents how much tilt omega profiles in a wave contain in its spatiotemporal evolution. Hence, we refer to $\tau_{r}$ and $\tau_{i}$ as the top-heaviness ratio and the tilt ratio, respectively. The name of the top-heaviness ratio is derived from Back et al. (2017), who assessed the topheaviness ratio of the climatological omega profiles.

\section{b. Demonstration}

Here we demonstrate how the top-heaviness ratio and the tilt ratio, defined in Eqs. (7)-(9), represent the 
(a) Top-heaviness $=0.3$ Tilt $=0.4$

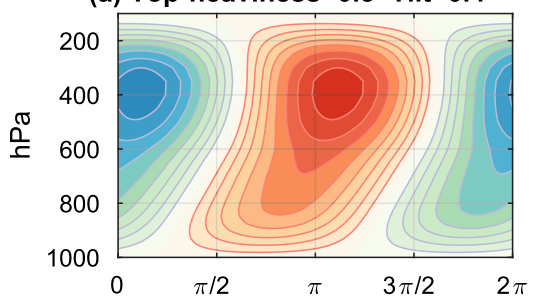

(d) Top-heaviness $=0$ Tilt $=0.4$

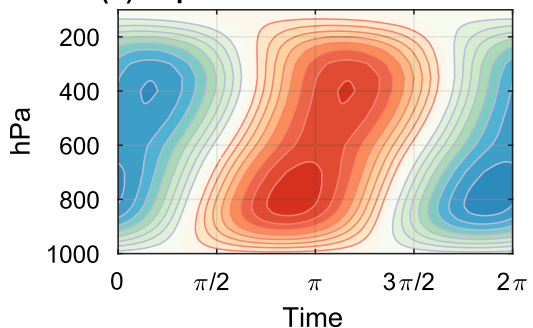

(b) Top-heaviness $=0.3$ Tilt $=0.2$

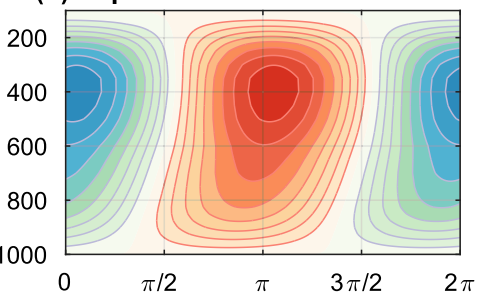

(e) Top-heaviness $=0$ Tilt $=0.2$

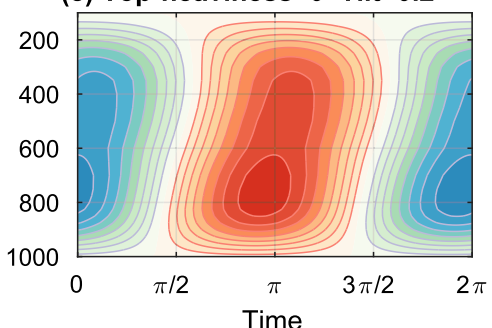

(c) Top-heaviness $=0.3$ Tilt $=0$

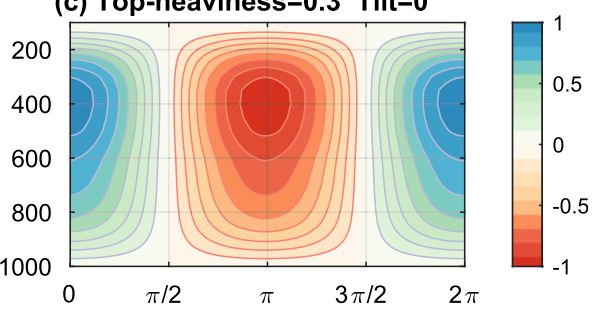

(f) Top-heaviness $=0$ Tilt $=0$

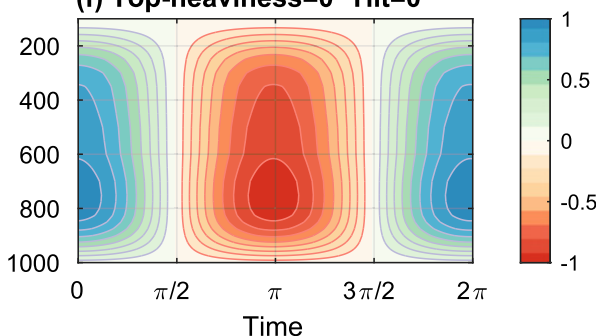

FIG. 2. Synthesized height-time structures of omega profiles constructed with Eq. (10), with different values of the top-heaviness ratio $\tau_{r}$ and the tilt ratio $\tau_{i}$. In the columns, the tilt ratio is decreased from (left) 0.4 to (center) 0.2 to (right) 0 , with the top-heaviness ratio fixed. In the rows, the top-heaviness ratio is decreased from (a)-(c) 0.3 to (d)-(f) 0 , with the tilt ratio fixed. The amplitude was normalized.

structures of the spatiotemporal evolution of omega profiles. To illustrate this, we synthesize an idealized time series of PC1 and PC2 with different values of the top-heaviness ratio and the tilt ratio, and reconstruct omega profiles with those.

For this demonstration, we create a time series of $\mathrm{PC} 1$ as the simplest wave, $\exp \{i(x-t)\}$, which is a wave with the zonal and temporal wavenumbers of the unit. Based on Eq. (7), we calculate the time series of PC2 as $\tau \exp \{i(x-t)\}$, where $\tau$ is a prescribed complex number. Then, from Eq. (5), the synthesized omega $\left(\omega_{\text {syn }}\right)$ profile is expressed as

$$
\omega_{\text {syn }}=\operatorname{Re}\left[\exp \{i(x-t)\} \Omega_{1}+\tau \exp \{i(x-t)\} \Omega_{2}\right],
$$

which is plotted in Fig. 2 with different values of $\tau$.

As the tilt ratio decreases with the top-heaviness ratio fixed (from left to right in each row of Fig. 2), the heighttime structures of the omega profiles become more vertically stacked, but the height of the maximum does not change. For instance, when the top-heaviness ratio is fixed at 0.3 in the top panels, the maximum upward (and downward) motion happens in the upper troposphere at around $400 \mathrm{hPa}$. In contrast, with the top-heaviness ratio of 0 in the bottom panels, the maximum (and the minimum) happens in the lower troposphere at around $800 \mathrm{hPa}$.

This figure demonstrates that the two important properties of omega profiles, tilts, and top-heaviness can be succinctly and quantitatively expressed with the tilt ratio and the top-heaviness ratio. By comparing those quantities among the different waves, we can answer the questions posed in section 1 . Inversely, if we know the values of the top-heaviness ratio and the tilt ratio, we can reconstruct the height-time (or height-longitude) structures of omega profiles as in Fig. 2.

\section{Results}

In this section, we show the top-heaviness and the tilt ratios in the zonal wavenumber and temporal frequency domain, and answer the questions posed in section 1 . Furthermore, we investigate the quantities associated with the moisture mode instability: 1) the GMS, 2) cloudradiation feedback, and 3) effective GMS. We also investigate the geographic dependency of our results.

Before showing these analyses, we must verify two assumptions: 1) The variables in ERA-I over the tropical oceans contain wave signals; and 2) the approximation of omega profiles with EOF1 and EOF2 is valid among all the waves.

\section{a. Wave signals in ERA-I over tropical oceans}

We first need to verify if the ECMWF model simulates the signals of all the tropical waves because the omega profiles in ERA-I are strongly constrained by the simulation skills-a more thorough discussion of the fidelity of the ERA-I data is given in section 5a. Second, we also need to test whether or not masking out the land data affects the spectral properties of the tropical waves. For doing those, we conduct a spectral analysis of the OLR (or the top thermal radiation) in the forecast data in ERA-I, and compare it with the observational OLR, with the zero padding over land. 
(a) Obs. OLR [Anti; Ocn; Adj. B.Noise]

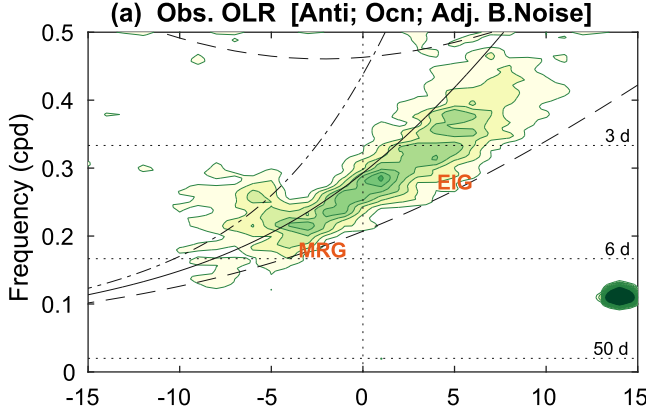

(c) ERAI OLR [Anti; Ocn; Adj. B.Noise]

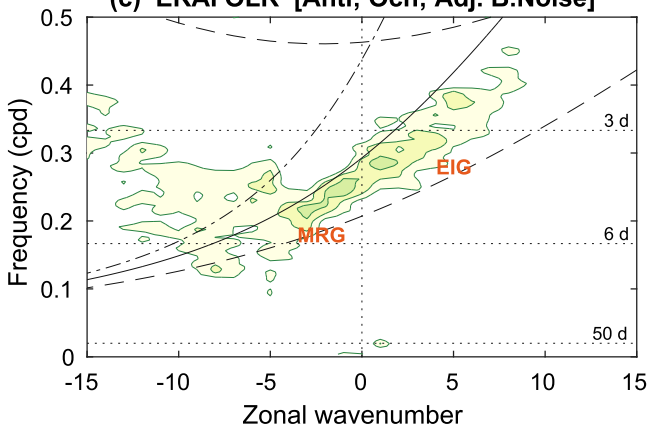

(b) Obs. OLR [Symm; Ocn; Adj. B.Noise]

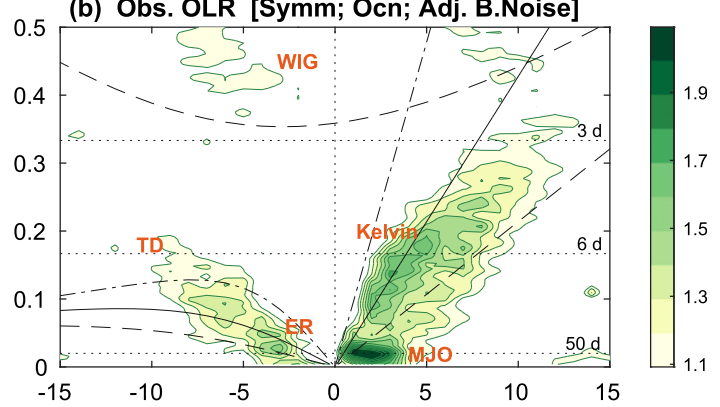

(d) ERAI OLR [Symm; Ocn; Adj. B.Noise]

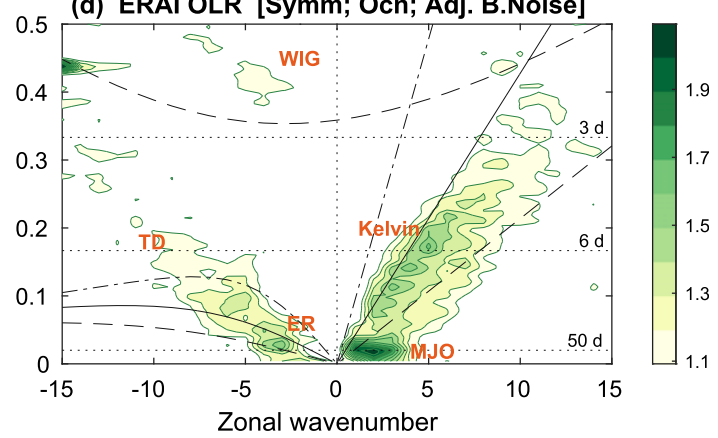

FIG. 3. The (left) antisymmetric and (right) symmetric components of the space-time spectral diagrams of the OLR in (a),(b) the satellite-observational data and (c),(d) ERA-I computed only over the oceans. The spectral signals are defined as the raw power divided by the adjusted background power, which is computed with the procedures described in appendix. The contours start from 1.092, a statistically significant threshold with a $90 \%$ significant level in a chi-squared test. The dispersion curves correspond to the equivalent depths of 10 (dashed), 40 (solid), and $200 \mathrm{~m}$ (dash-dotted line).

Figure 3 is a normalized space-time spectral diagram (aka Wheeler-Kiladis diagram; after Wheeler and Kiladis 1999). The power spectra smoothed with three passes of a 1-2-1 running-mean filter in the temporal frequency dimension-as described in section $2 \mathrm{~d}$-are divided by the background power spectra. The background power spectra were estimated similarly to Wheeler and Kiladis (1999). However, for comparing the OLR in the observation with that in ERA-I, some adjustment of the background power spectra is required. Since this adjustment process is not related to the main results of this paper, the details of it are described in appendix.

In Fig. 3, the contour starts from 1.092, the threshold for statistical significance in a chi-squared test with a $90 \%$ significance level and 396 degrees of freedom (DOF). The DOF was estimated as 2 (amplitude and phase) $\times 3\left(1-2-1\right.$ running-mean filter $\left.{ }^{2}\right) \times 66$ (number of time series segments).

\footnotetext{
${ }^{2}$ We have no consensus regarding the number of the DOF contribution due to the 1-2-1 running-mean filter. For instance, Yasunaga et al. (2019) assumed it to be 2 instead of 3. However, that difference does not affect the main results in this study.
}

The spectral signals in the OLR simulated by the ECMWF model (Figs. 3c,d) are comparable to those in the observational data (Figs. 3a,b) - with some underestimations pointed out by Kim et al. (2014b). Additionally, Fig. 3, which masked out the land data, is comparable to Fig. A2 which includes the land data-except for slight underestimations at higher frequencies. Therefore, this result verifies that ERA-I possesses the signals of all the tropical waves, and that the exclusion of the land data does not affect the spectral properties in a way that impacts the conclusions of this study.

\section{b. Fraction of variance explained by EOF-PC pairs}

Next, we examine the validity of the approximation of omega profiles with the two leading EOFs as in Eq. (5). Although we know that the first two leading EOF-PC pairs explain $83.5 \%$ of the total spatiotemporal variance of the omega profiles over the tropical oceanic belt, it does not have to be the case among all the waves. It might be possible that omega profiles in some waves are composed of other EOFs.

For examining it, we compute the fraction of the variance explained by the two leading EOF-PC pairs in the zonal-wavenumber and temporal-frequency domain. In general, the fraction of the variance explained by the $j$ th EOF-PC pair is defined as 


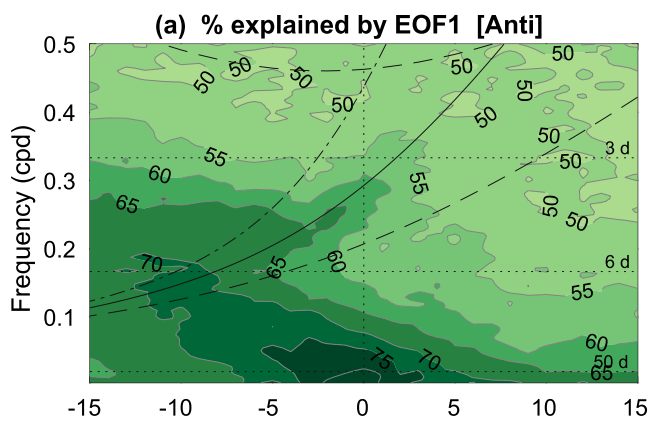

(c) \% explained by EOF2 [Anti]

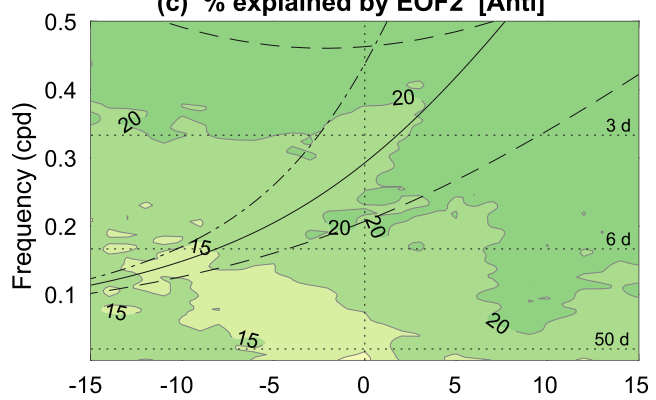

(e) \% explained by EOF1+2 [Anti]

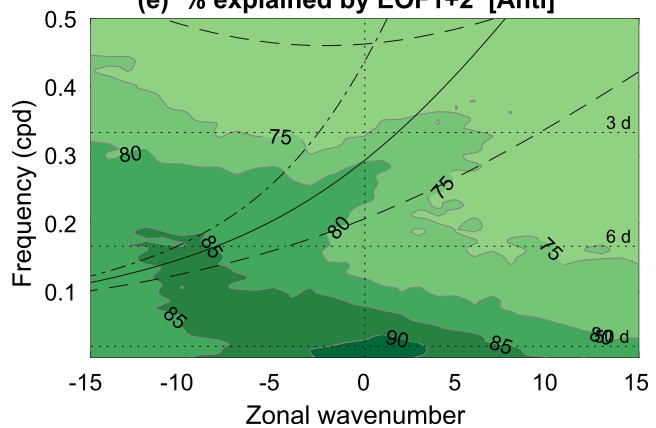

(b) \% explained by EOF1 [Symm]

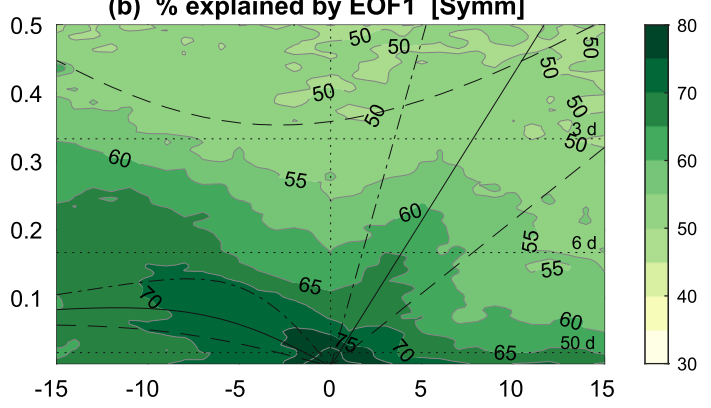

(d) \% explained by EOF2 [Symm]

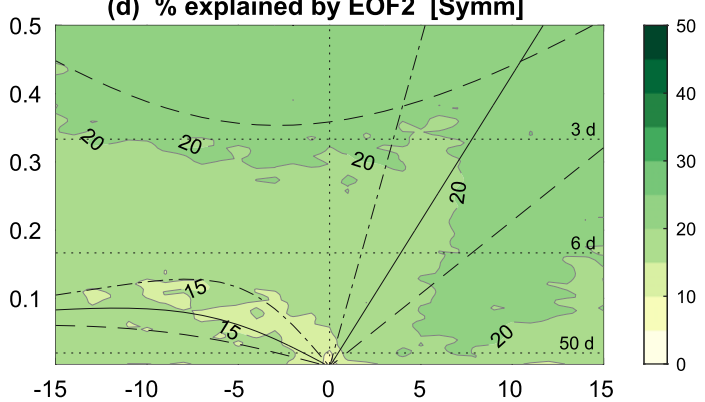

(f) $\%$ explained by EOF1+2 [Symm]

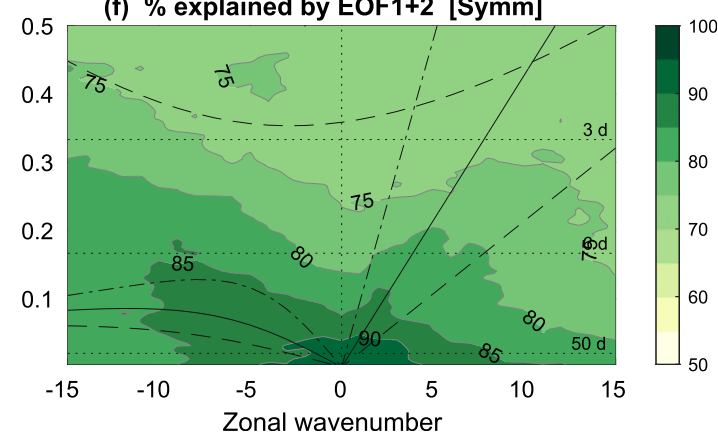

FIG. 4. The fractions (\%) of the spatiotemporal variance of omega profiles explained by (a),(b) the EOF1-PC1 pair, (c),(d) EOF2-PC2 pair, and (e),(f) both the EOF1-PC1 and EOF2-PC2 pairs for the (left) antisymmetric and (right) symmetric components. The fractions are computed with Eq. (13). The contour line (and color scale) in each row starts from a different value, but the contour interval is the same (5\%). Dispersion curves are as in Fig. 3.

$$
\frac{\sum_{m=1}^{M} \sum_{n=1}^{N} o_{j}^{2}\left(x_{m}, t_{n}\right)}{\sum_{m=1}^{M} \sum_{n=1}^{N}\left\{o_{1}^{2}\left(x_{m}, t_{n}\right)+o_{2}^{2}\left(x_{m}, t_{n}\right)+\cdots+o_{J}^{2}\left(x_{m}, t_{n}\right)\right\}},
$$

where $J$ is the total number of the EOF-PC pairs, or the number of the vertical levels (in this study, $J=27$ ). From Parseval's theorem [Eq. (A1)], this is equivalent to

$$
\frac{\sum_{k=1}^{M} \sum_{l=1}^{N}\left|O_{j}(k, l)\right|^{2}}{\sum_{k=1}^{M} \sum_{l=1}^{N}\left\{\left|O_{1}(k, l)\right|^{2}+\left|O_{2}(k, l)\right|^{2}+\cdots+\left|O_{J}(k, l)\right|^{2}\right\}},
$$

where $O_{j}$ is the Fourier transformation of the $j$ th $\mathrm{PC}$, as defined in Eq. (6). Therefore, for a single wave component with a fixed zonal wavenumber $k$ and temporal frequency $l$, the fraction of the variance explained by the $j$ th EOF-PC pair can be formally defined as

$$
R_{j}(k, l) \equiv \frac{\left|O_{j}(k, l)\right|^{2}}{\left|O_{1}(k, l)\right|^{2}+\left|O_{2}(k, l)\right|^{2}+\cdots+\left|O_{J}(k, l)\right|^{2}},
$$

The fractions for the first and second leading pairs $R_{1}$ and $R_{2}$ were computed with the procedures described in section $2 \mathrm{~d}$.

Figures $4 \mathrm{a}$ and $4 \mathrm{~b}$ show that $R_{1}$ is greater than $\sim 50 \%$ over the entire wavenumber-frequency domain of interest for both the antisymmetric and symmetric 
components. Furthermore, as the temporal frequency decreases, $R_{1}$ increases, reaching $70 \%-75 \%$ on the MJO and ER wave scales. This result shows that the fraction of the variance explained by the first EOF-PC pair has a strong scale dependency. It also has a skewness in the direction of the zonal wavenumber. In general, westward-propagating disturbances with negative zonal wavenumbers have higher values of $R_{1}$, which suggests that the omega profiles in the westward-propagation disturbances-including both the wave phenomena and nonwave disturbances - are predominantly explained by the first EOF-PC pair.

In contrast, Figs. $4 \mathrm{c}$ and $4 \mathrm{~d}$ show that the scale dependency of $R_{2}$ is small; $R_{2}$ is relatively homogeneous (about $20 \%$ ) over the entire wavenumber-frequency domain of interest. It does, however, slightly decrease as the frequency decreases. That is opposite to the pattern seen in $R_{1}$.

By adding $R_{1}$ and $R_{2}$, we can calculate how much of the variance of the omega profiles in ERA-I is explained by the first two leading EOF-PC pairs in the wavenumber-frequency domain. Figures $4 \mathrm{e}$ and $4 \mathrm{f}$ show that the first two leading pairs explain at least $75 \%$ of the variance of the omega profiles among all the waves depicted in Fig. 3, and the fraction increases as the time scale gets longer. On the ER scale, it accounts for $85 \%-$ $90 \%$. On the MJO scale, it accounts for $>90 \%$. Even on the WIG scale, $>75 \%$ of the variance is explained by the two leading EOF-PC pairs. It has a skewness in the direction of the zonal wavenumber, which comes from the skewness of $R_{1}$ discussed above. In general, the omega profiles in the westward-propagating disturbances are more explained by the two leading pairs than those in the eastward-propagating disturbances are.

The results above verify that the approximation of omega profiles with the two leading EOFs, as in Eq. (5), is valid among all the waves. The two leading EOF-PC pairs explain most of the variability of the omega profiles-greater than 75\%-among all the waves, and it becomes more valid as the time scale gets longer.

It should be noted that Fig. 4 represents the structure of omega profiles in all kinds of tropical disturbances, including not only the wave phenomena, but also nonwave phenomena which are generally considered as background noise. For instance, Figs. 4a, 4b, 4e, and $4 \mathrm{f}$ show some dominant signals in the negative zonal wavenumbers, which are found commonly between the antisymmetric and symmetric components. These signals may originate not only from the TD-type waves, but also from ubiquitous convection advected by the background easterly wind, whose meridional structures are neither symmetric nor antisymmetric-convective clusters in or skewed to one hemisphere.

\section{c. Top-heaviness and tilt ratios}

Here we show the results of the top-heaviness ratio and the tilt ratio, defined in Eqs. (7)-(9). Since the approximation of omega profiles with EOF1 and EOF2 is valid among all the waves of interest, the values of the top-heaviness ratio and the tilt ratio succinctly represent the structures of the spatiotemporal evolution of the omega profiles in each wave as demonstrated in Fig. 2.

Figures $5 \mathrm{a}$ and $5 \mathrm{~b}$ show the top-heaviness ratio of the antisymmetric and symmetric component, respectively. The reddish color indicates the omega profiles are topheavy at the convective maximum as demonstrated in Fig. 2. In general, omega profiles become more topheavy as the time and spatial scales get longer and larger; and the MJO has the most top-heavy profile while the WIG and EIG waves have the most bottomheavy profile among all the waves. The ER and lowfrequency Kelvin waves also have top-heavy profiles, but not as top-heavy as the MJO. This result is at odds with $\mathrm{K} 11$, who claimed that the MJO has the most bottom-heavy omega profile that may be responsible for the spatial scale selection of the MJO.

The Kelvin wave has a wide range of top-heaviness ratios. The high-frequency Kelvin wave (3-4-day periodicity) has a top-heaviness ratio close to 0 whereas the low-frequency Kelvin wave ( $\sim 10$-day periodicity or $0.1 \mathrm{cpd}$ ) has about 0.25 .

Figures $5 \mathrm{c}$ and $5 \mathrm{~d}$ show the tilt ratio for the antisymmetric and symmetric components, respectively. The high-frequency Kelvin wave with $\sim 0.25 \mathrm{cpd}$ and the EIG wave have the most tilted omega profiles in their spatiotemporal evolution. Meanwhile, the ER wave has the most vertically stacked profiles with a tilt ratio close to 0 (or even slightly negative). In the continuum from the Kelvin wave to the MJO, the tilt ratio decreases, and thus, the MJO has a smaller value of the tilt ratio than the other waves excluding the ER wave. Therefore, this diagnosis indicates that the ER wave and the MJO have more vertically stacked omega profiles than the other waves including the Kelvin, WIG, EIG, and MRG waves which exhibit significant tilts.

Although the tilt ratio varies continuously in the wavenumber-frequency domain, we observe a distinct separation between the fast-moving CC gravity wave modes and the moisture modes. The wave members categorized as the fast-moving CC gravity wave modes (summarized in Table 1) exhibit significant tilts in the omega profiles while the ER wave and the $\mathrm{MJO}$, which theoretically belong to the slow-moving moisture modes, have more vertically stacked structures. The TD-type wave, categorized as a slow-moving mode, does not have a distinct pattern of the tilt ratio; it has a range from 

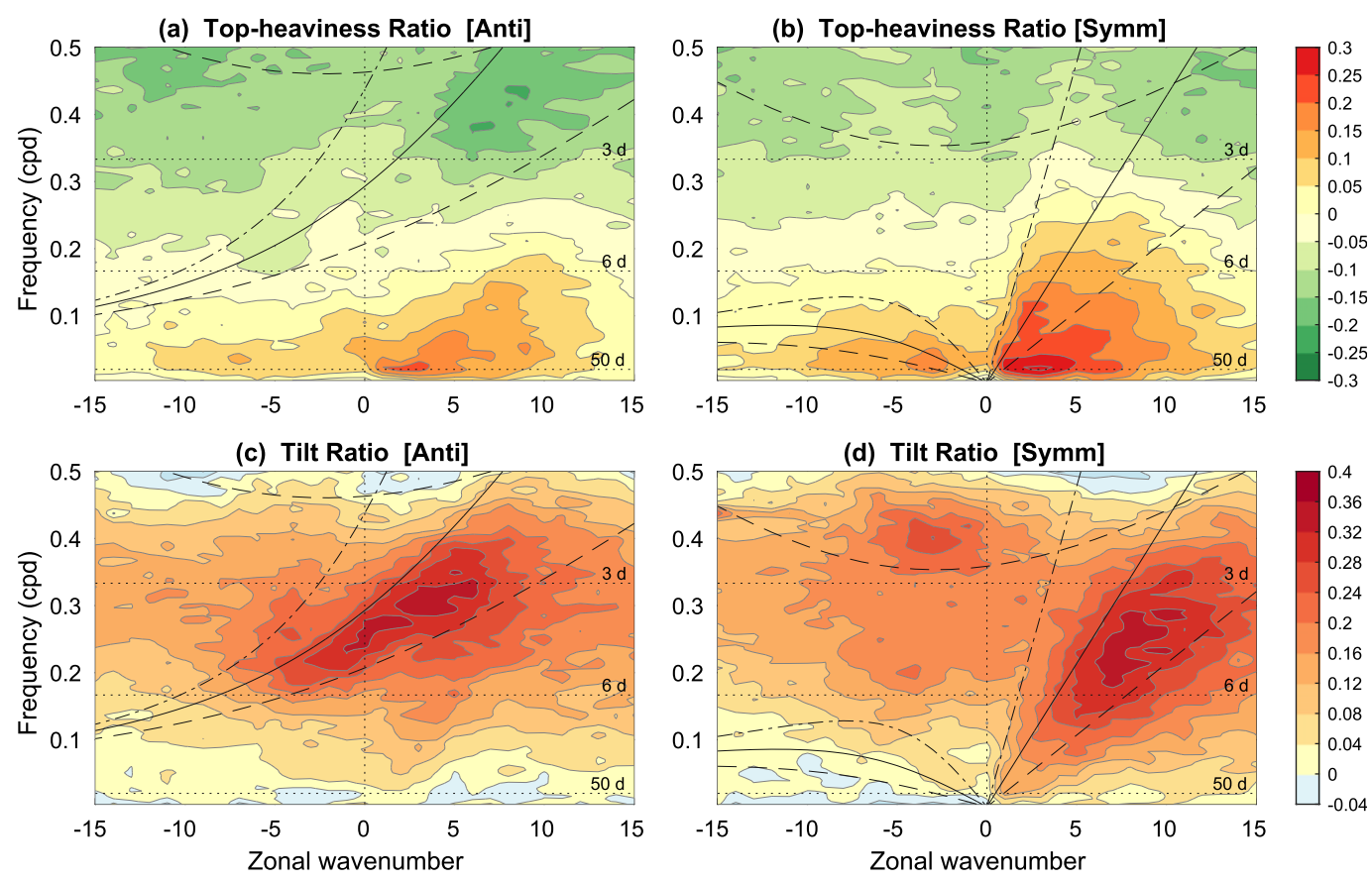

FIG. 5. (a),(b) The top-heaviness ratio $\tau_{r}$ and (c),(d) the tilt ratio $\tau_{i}$, defined in Eqs. (7)-(9) for the (left) antisymmetric and (right) symmetric components. The dispersion curves are as in Fig. 3.

0.04 to 0.16 in the symmetric component. In the antisymmetric component, the TD-type wave generally overlaps with the MRG wave which has a significant tilt (e.g., Kiladis et al. 2009). Thus, the tilting structures of the TD-type wave-referred to as easterly waves-pointed out by Reed and Recker (1971) might be those of the MRG wave.

The tilt ratio also exhibits a strong scale dependency. Roughly speaking, the tilt becomes smaller as the time scale gets longer as speculated by Inoue and Back (2015a). However, the transition of the tilt ratio is not monotonic. It is complicated with a strong dependency on the wave type as shown in Figs. 5c and 5d.

As in the top-heaviness ratio, the Kevin wave has a wide range of tilt ratios, ranging from $\sim 0.18$ for the lowfrequency Kelvin wave to $\sim 0.32$ for the high-frequency Kelvin wave. Therefore, we recommend that the Kelvin wave be separated into a high-frequency and low-frequency component when investigated for its structures.

The gradual changes in the top-heaviness and tilt ratios from the Kelvin wave to the MJO might indicate a continuum between the Kelvin wave and the MJO, as suggested by previous diagnostic studies (e.g., Roundy 2012a,b) and by a theoretical hypothesis (e.g., Adames et al. 2019).

For facilitating the comparison of the top-heaviness and the tilt ratios among the different waves, these values are summarized in Table 2 for the different waves. Each wave is defined with a box of wavenumbers and frequencies. For instance, the MJO is defined with the box of $1 \leq k \leq 5$ and $1 / 100 \leq$ freq $\leq 1 / 30$ (cpd), where $k$ is a zonal wavenumber and freq is a temporal frequency. And with the corresponding top-heaviness and tilt ratios, we reconstructed the height-time structures of the omega profiles in Fig. 6 with Eq. (10) for each wave.

In summary, the MJO (Fig. 6a), ER (Fig. 6b), and lowfrequency Kelvin (Fig. 6c) waves have top-heavy omega profiles at the convective maximum when the phase is $\pi$. The peak of ascent happens in the upper troposphere at around $400 \mathrm{hPa}$. In contrast, the WIG (Fig. 6e), EIG (Fig. 6f), and MRG (Fig. 6g) waves have bottom-heavy omega profiles at the convective maximum; the peak of ascent happens in the lower troposphere at around $800 \mathrm{hPa}$. The MJO and ER wave have more vertically stacked omega profiles compared to the high-frequency Kelvin, WIG, EIG, and MRG waves, which have significant tilts. The Kelvin waves have a wide range of topheaviness and tilts; the high-frequency Kelvin wave (Fig. 6d) has a more bottom-heavy, tilted omega profile compared with the low-frequency Kelvin wave (Fig. 6c), which has a more top-heavy, vertically stacked profile.

\section{d. GMS, cloud-radiation feedback, and effective GMS}

According to the previous moisture mode theories (e.g., Sobel and Gildor 2003; Fuchs and Raymond 2007; 
TABLE 2. Summary of the top-heaviness ratio $\tau_{r}$ and the tilt ratio $\tau_{i}$ for each wave defined by a different box of wavenumbers and frequencies.

\begin{tabular}{lccr}
\hline \hline \multicolumn{1}{c}{ Wave name } & Wavenumbers & Frequencies (cpd) & $\tau_{r}$ \\
\hline MJO & {$[1,5]$} & {$[1 / 100,1 / 30]$} & 0.261 \\
ER & {$[-4,-3]$} & {$[1 / 50,1 / 15]$} & 0.150 \\
Low-frequency Kelvin & {$[2,3]$} & {$[1 / 14,1 / 10]$} & 0.087 \\
High-frequency Kelvin & {$[5,7]$} & {$[1 / 5,1 / 4]$} & 0.043 \\
WIG & {$[-5,-3]$} & {$[1 / 2.5,1 / 2.2]$} & 0.180 \\
EIG & {$[1,3]$} & {$[1 / 4,1 / 3]$} & -0.094 \\
MRG & {$[-3,-1]$} & {$[1 / 5,1 / 4]$} & -0.075 \\
\hline
\end{tabular}

Raymond and Fuchs 2007; Sobel and Maloney 2013; Sugiyama 2009; Adames and Kim 2016; Fuchs and Raymond 2017), there are three important parameters for the instability of the moisture modes: 1) the gross moist stability (GMS), 2) the cloud-radiation feedback, and 3) the effective GMS. The effective GMS is a measure of the GMS that includes the impact of the cloud-radiation feedback on the moisture modes. In this subsection, these three parameters are computed on the wavenumber-frequency domain.

Following Inoue and Back (2015b), the (vertical) GMS can be defined as a real number $\Gamma_{v}$ that is written as

$$
\Gamma_{v} \equiv \frac{[\langle\omega \partial h / \partial p\rangle\langle\omega \partial s / \partial p\rangle]}{\left[\langle\omega \partial s / \partial p\rangle^{2}\right]}
$$

where $\omega$ is vertical pressure velocity (omega), $s \equiv C_{p} T+$ $g z$ is dry static energy (DSE), enthalpy $C_{p} T$ plus geopotential height $g z$, and $h \equiv s+L q$ is moist static energy (MSE), the DSE plus total latent heat $L q$. The angle brackets represent a mass-weighted vertical integration from the surface pressure to $100 \mathrm{hPa}$, and the square brackets represent an average over time and zonal space.
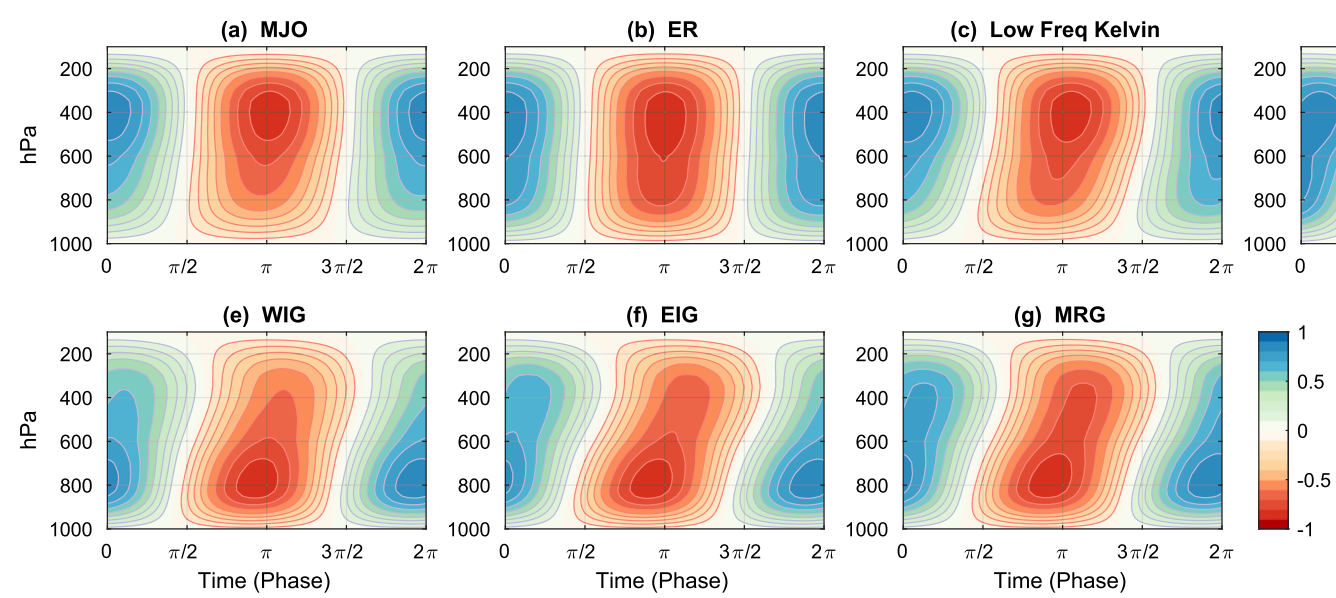

FIG. 6. Reconstructed height-time structures of omega profiles for (a) the MJO, (b) ER, (c) low-frequency Kelvin, (d) high-frequency Kelvin, (e) WIG, (f) EIG, and (g) MRG waves with their corresponding top-heaviness ratios $\tau_{r}$ and tilt ratios $\tau_{i}$. The values of $\tau_{r}$ and $\tau_{i}$ and the spectral regions of each wave are summarized in Table 2. The amplitude was normalized as in Fig. 2.
Similarly, the cloud-radiation feedback parameter can be defined as a real number $r$ that is written as

$$
r \equiv \frac{\left[\left\langle Q_{R}\right\rangle\langle\omega \partial s / \partial p\rangle\right]}{\left[\langle\omega \partial s / \partial p\rangle^{2}\right]},
$$

where $\left\langle Q_{R}\right\rangle$ is column-integrated radiative heating, which is estimated as top net radiation (top thermal radiation plus top solar radiation) minus surface net radiation (surface thermal radiation plus surface solar radiation) in the ERA-I datasets. The cloudradiation feedback parameter represents the reduction of radiative cooling due to the greenhouse effect of clouds (e.g., Su and Neelin 2002; Bretherton and Sobel 2002; Lin and Mapes 2004; Peters and Bretherton 2005; Kim et al. 2015). All the column-integrated quantities in Eqs. (14) and (15) correspond to temporal anomalies.

Finally, the effective GMS $\Gamma_{e}$ is defined as

$$
\Gamma_{e} \equiv \Gamma_{v}-r
$$

Although there are some variations in their definitions, the concepts of the GMS, cloud-radiation feedback, and
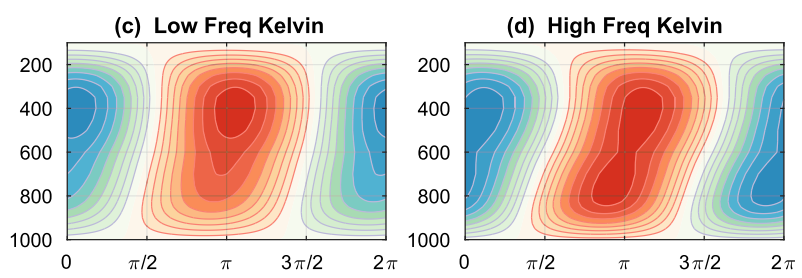
effective GMS are the same among all the moisture mode theories.

For the growth (or the instability) of the moisture modes, a low ${ }^{3}$ value of $\Gamma_{e}$ is necessary. But Fig. 5 shows that the omega profile in the MJO is the most top-heavy among all the waves, which is generally associated with a high value of $\Gamma_{v}$ that increases $\Gamma_{e}$. Then, how would the instability of the moisture modes happen on the MJO scale? To answer this question, we computed $\Gamma_{v}, r$ and $\Gamma_{e}$ in the wavenumber-frequency domain.

The computation of these quantities is the same as that of the top-heaviness ratio. In general, we can compute a real constant $c$ in the form of

$$
c=\frac{[a(x, t) b(x, t)]}{\left[b(x, t)^{2}\right]}
$$

in the wavenumber-frequency domain, as

$$
C(k, l)=\frac{\operatorname{Re}\{\mathscr{F}\{a(x, t)\} \mathscr{F} *\{b(x, t)\}\}}{\mathscr{F}\{b(x, t)\} \mathscr{F} *\{b(x, t)\}},
$$

where $\mathrm{Re}$ is a real component of a complex number, $\mathscr{F}$ is the Fourier transformation, and the star represents the complex conjugate. In other words, $C$ is the cospectrum of $a$ and $b$ divided by the power spectrum of $b$. One can replace $a$ and $b$ with the variables in Eqs. (14) and (15) to obtain $\Gamma_{v}, r$, and $\Gamma_{e}$ in the wavenumberfrequency domain. For computing the fractional quantities, we follow the procedures described in section $2 \mathrm{~d}$. A similar method has been employed by Yasunaga et al. (2019) and Adames et al. (2019). Especially, Yasunaga et al. (2019) computed similar quantities to $\Gamma_{v}$ and $r$; but because they used precipitation instead of $\langle\omega \partial s / \partial p\rangle$ in Eqs. (14) and (15), their results do not have to be the same, especially on short time scales where $\langle\omega \partial s / \partial p\rangle$ may not be approximated by precipitation.

Figures $7 \mathrm{a}$ and $7 \mathrm{~b}$ show the (vertical) GMS $\Gamma_{v}$ in the wavenumber-frequency domain. If $\Gamma_{v}$ was completely determined by the shape of the omega profiles, Figs. 7a and $7 \mathrm{~b}$ should look similar to Figs. 5a and 5b. However, the patterns of $\Gamma_{v}$ are more complicated than those of the top-heaviness ratio. For instance, the MJO with the most top-heavy omega profile does not exhibit the highest value of $\Gamma_{v}$. The highest value occurs in the fast-propagating Kelvin wave between the dispersion lines of 40- and 200-m equivalent depths. This fact indicates that MSE profiles also play an important role in setting $\Gamma_{v}$. Nevertheless, the overall pattern of

\footnotetext{
${ }^{3}$ If the wind-induced surface heat exchange (WISHE) effect is ignored, a negative $\Gamma_{e}$ is necessary.
}

$\Gamma_{v}$ in the wavenumber-frequency domain is largely consistent with that of the top-heaviness ratio. The value of $\Gamma_{v}$ on the MJO scale is relatively high $(0.12-0.14)$ compared to the other waves, with a few exceptions like the fast-propagating Kelvin wave. Therefore, Fig. 7b may reject the hypothesis by $\mathrm{K} 11$, who suggested that MJO-scale disturbances have a lower value of the GMS than the other scales.

While $\Gamma_{v}$ is relatively high on the MJO scale, the effective GMS $\Gamma_{e}$-depicted in Figs. $7 \mathrm{e}$ and $7 \mathrm{f}$ - exhibits a negative value on that scale, which are considered to favor the growth of the moisture modes. This negative $\Gamma_{e}$ is a result of a large value of the cloud-radiation feedback $r$, depicted in Figs. 7c and 7d. The value of $r$ increases toward longer time scales and larger spatial scales. This increase in $r$ (as a function of the scales) exceeds the increase in $\Gamma_{v}$, yielding negative values of $\Gamma_{e}$, or $\Gamma_{v}-r$.

Interestingly, the patterns of $\Gamma_{e}$ in Figs. $7 \mathrm{e}$ and $7 \mathrm{f}$ are linked to the categorization of the CCEWs and the MJO. The MJO, ER, and TD-type waves, which are categorized as the slow-moving modes (see Table 1), have negative values of $\Gamma_{e}$ whereas the Kelvin and WIG waves, which belong to the fast-moving modes, have positive values of $\Gamma_{e}$. This result may be linked to the different mechanisms of the instabilities of the CC gravity wave modes and the moisture modes (e.g., Raymond and Fuchs 2007; Fuchs et al. 2012). The exceptions are the EIG and MRG waves, which are categorized as the fast-moving modes, but have negative values of $\Gamma_{e}$.

Figure 7 shows the signals of all tropical disturbances including not only the CCEWs and MJO, but also other disturbances such as dry (or free) waves and neutral or damped modes. That is why Figs. 7a, 7b, 7e, and 7f, show the signals in the spectral regions not corresponding to the CCEWs and MJO (compared with Fig. 3). For instance, Fig. 7f shows the signals of dry Kelvin waves and dry EIG waves around the 200-m dispersion line.

We can also observe high (effective) GMS below the 10-m dispersion line in the positive zonal wavenumbers (Figs. 7b and 7f), which are not explained by the CCEW's spectra. Those signals might correspond to neutral or damped Kelvin waves. For instance, in the limit of the strict quasi-equilibrium (SQE) where temperature profiles are relaxed back to moist adiabatic profiles rapidly, there exists a neutral or slightly damped mode, which shares many common features with the convectively coupled Kelvin wave (e.g., Emanuel et al. 1994; Neelin and Yu 1994). Furthermore, Adames et al. (2019) suggested that there exist a hybrid of the Kelvin wave mode and the moisture mode. We speculate that the figures of the (effective) GMS capture the signals of 

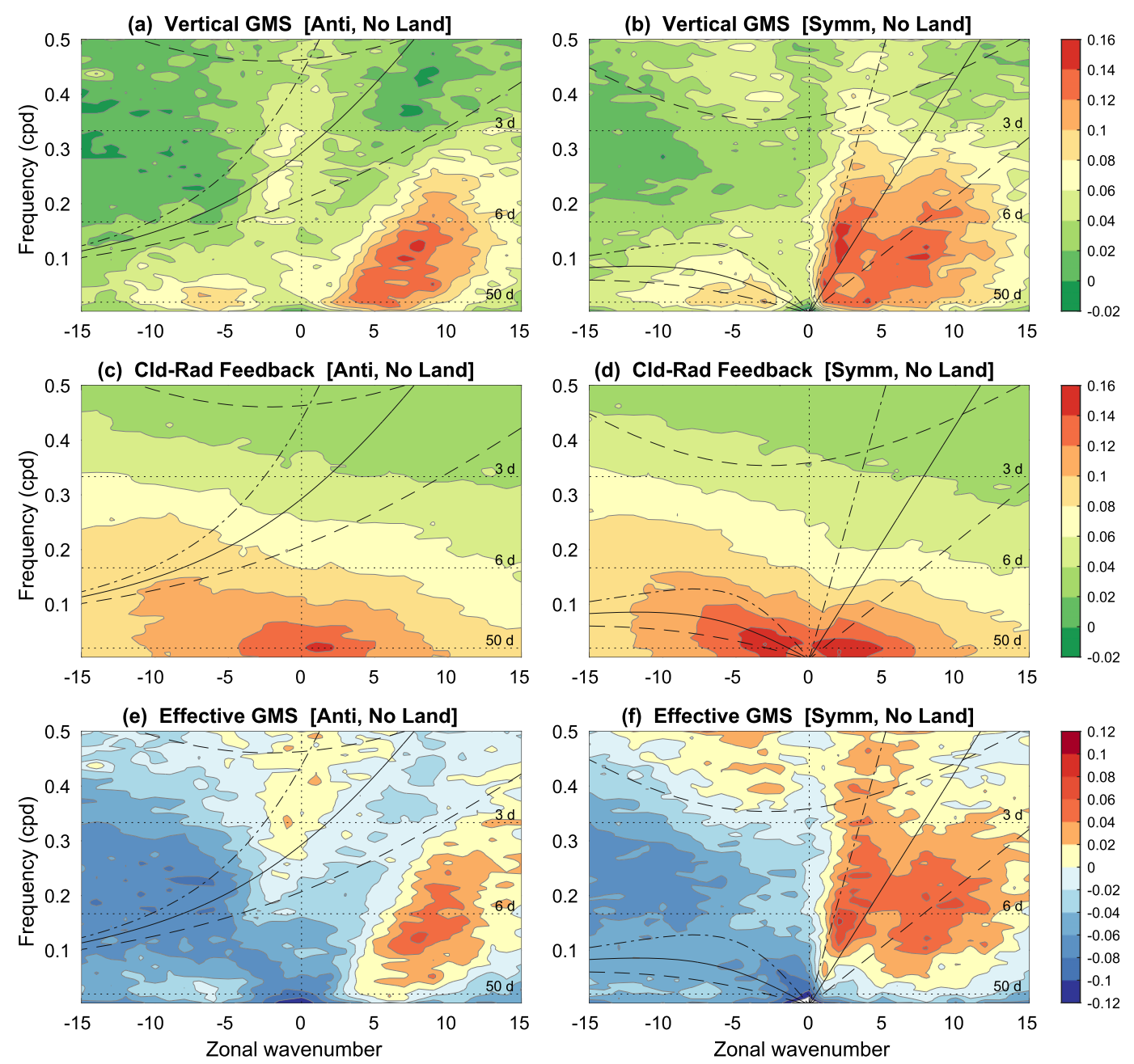

FIG. 7. (a),(b) The (vertical) GMS $\Gamma_{v}$, (c),(d) the cloud-radiation feedback $r$, and (e),(f) the effective GMS $\Gamma_{e}$. The quantities are defined in Eqs. (14)-(16), respectively, and computed with Eq. (18). The column show the (left) antisymmetric and (right) symmetric components. The dispersion curves are as in Fig. 3.

those modes, which are generally considered as background noise. Since the location of the Kelvin waves is usually skewed to the Northern Hemisphere where the ITCZ is located, we can also observe the signals of the damped Kelvin waves in the antisymmetric component (Figs. 7a,e). These antisymmetric signals disappear in the regional spectra computed over the warm pool region where the ITCZ is more symmetric around the equator (shown in the next).

\section{e. Geographic variability of top-heaviness}

The shape of omega profiles varies significantly among different oceanic basins. Specifically, climatological omega profiles in the eastern Pacific and the Atlantic Ocean are bottom-heavy whereas those in the warm pool region including the western Pacific and the Indian Ocean are top-heavy (e.g., Back and
Bretherton 2006, 2009; Back et al. 2017). In this subsection, we investigate how much this geographic variability affects our diagnoses.

As in Back et al. (2017), we calculated the topheaviness ratio over the tropical oceans. We can write the top-heaviness ratio $\widetilde{\tau_{r}}$ as

$$
o_{2}(x, y, t)=\widetilde{\tau}_{r} o_{1}(x, y, t),
$$

where the tilde, used for distinguishing from $\tau_{r}$ in the Fourier space, represents a value in the longitudelatitude space. Back et al. (2017) calculated $\widetilde{\tau_{r}}$ as a ratio of the time average of $o_{2}$ to the time average of $o_{1}$. In this study, we calculated it as a regression slope:

$$
\widetilde{\tau}_{r}=\frac{\overline{o_{1}^{\prime} o_{2}^{\prime}}}{\overline{o_{1}^{\prime 2}}},
$$



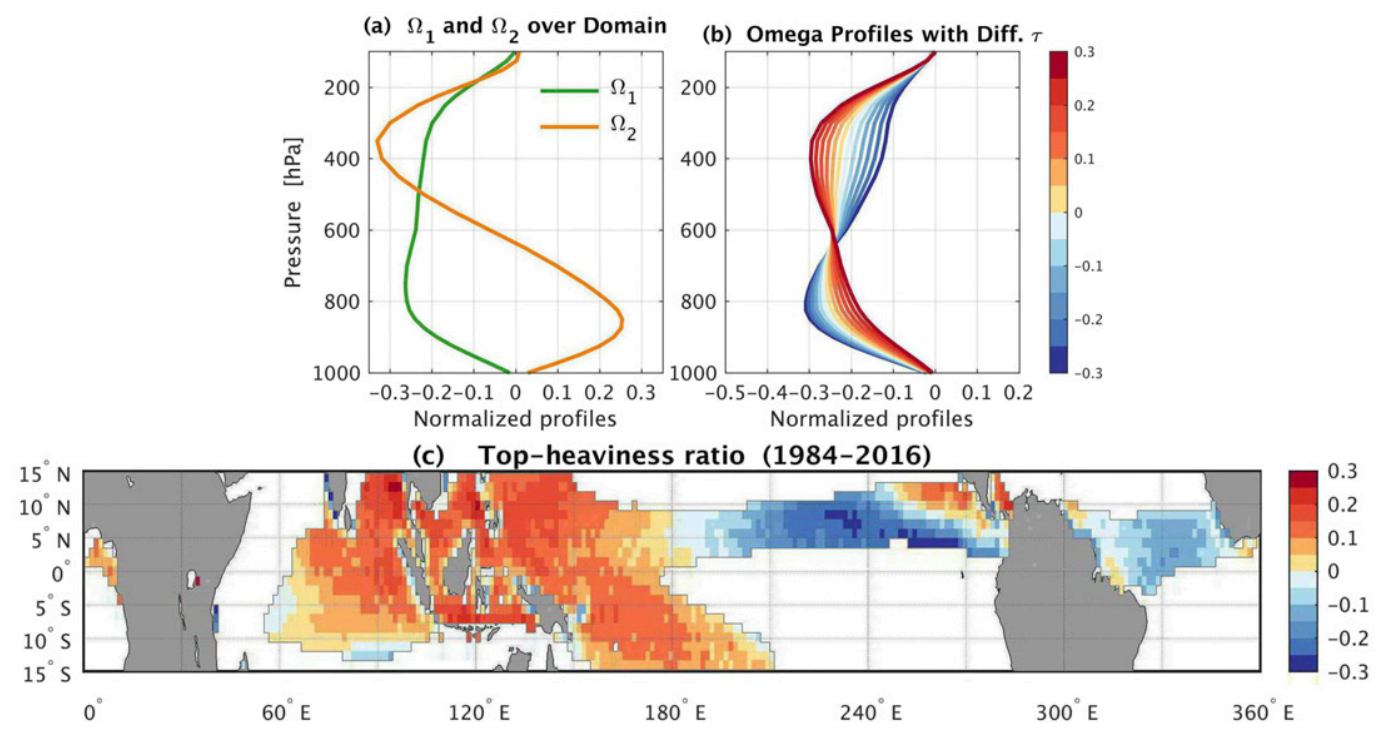

FIG. 8. (a) As in Fig. 1. (b) Omega profiles with different top-heaviness ratios ranging from -0.3 to 0.3. (c) Map of the top-heaviness ratio, plotted only over the regions where the mean $o_{1}$ is greater than $0.05 \mathrm{~Pa} \mathrm{~s}^{-1}$. The color scales in (b) and (c) are the same.

where the prime represents a temporal anomaly, and the bar represents a time average.

Figure 8 shows the geographic patterns of the topheaviness ratio, calculated with Eq. (20). We plotted it only over the regions where $\overline{o_{1}}$ is greater than $0.05 \mathrm{~Pa} \mathrm{~s}^{-1}$; the masked regions do not have significant convective activities, thus contribute little to the spectral analyses. The value of the top-heaviness ratio can be compared to Fig. $8 \mathrm{~b}$ for its corresponding vertical shape. As Back et al. (2017) showed, the omega profiles are top-heavy in the warm pool region while they are bottom-heavy in the eastern Pacific and the Atlantic Ocean-excluding the Mesoamerican region where the omega profiles are top-heavy.

Then, how much does this geographic variability in the top-heaviness ratio affect the conclusions obtained from Figs. 5 and 7? To answer this question, we reproduced these figures with regional spectral analyses. Since one of our primary interests is the MJO, we computed regional spectra within the zonal section of the warm pool region ranging from $60^{\circ} \mathrm{E}$ to $180^{\circ}$, using the zonal tapering method discussed in section $2 \mathrm{c}$.

Figure 9 shows the top-heaviness and tilt ratios in the warm pool region. There are some discernible differences from Fig. 5. Over the warm pool region, the omega profiles are more top-heavy among the entire frequencywavenumber domain, compared with the global topheaviness figure (Figs. 5a,b), which is not surprising because of the high top-heaviness ratios over the warm pool region (Fig. 8c). As a result, the MJO and ER wave over the warm pool region exhibit significantly high values of the top-heaviness ratio. The TD-type wave there also exhibits higher values of the top-heaviness ratio than the global values. The regional tilt ratio (Figs. 9c,d) exhibits similar patterns to the global tilt ratio (Figs. 5c,d) except for slightly higher tilt ratios in the Kelvin and WIG waves over the warm pool region.

Figure 10 shows the GMS, cloud-radiation feedback, and effective GMS over the warm pool region. Because of the top-heavy omega profiles over the warm pool region, the GMS there is slightly higher especially at high frequencies (Figs. 10a,b) compared to the GMS over the globe (Figs. 7a,b). Additionally, unlike the global GMS figure, we can observe distinct high-value signals of the GMS in the Kelvin wave and MJO in the regional GMS figure. Furthermore, because the ITCZ position is more symmetric around the equator in the warm pool region, there are no antisymmetric Kelvin wave signals in Fig. 10a, unlike Fig. 7a. The regional cloud-radiation feedback in the ER wave and MJO is higher (Figs. 10c,d) than the global one (Figs. 7c,d). And unlike Fig. 7e, the EIG and MRG waves exhibit positive effective GMS in Fig. 10e. Therefore, in the warm pool region, the moisture modes including the MJO, ER, and TD-type waves exhibit negative effective GMS whereas the CC gravity waves including the Kelvin, WIG, EIG, and MRG waves exhibit positive effective GMS. That may be linked to different mechanisms of the instabilities of the CC gravity wave modes and the moisture modes.

Despite those differences in the regional analyses, they do not affect the primary conclusions we obtained 


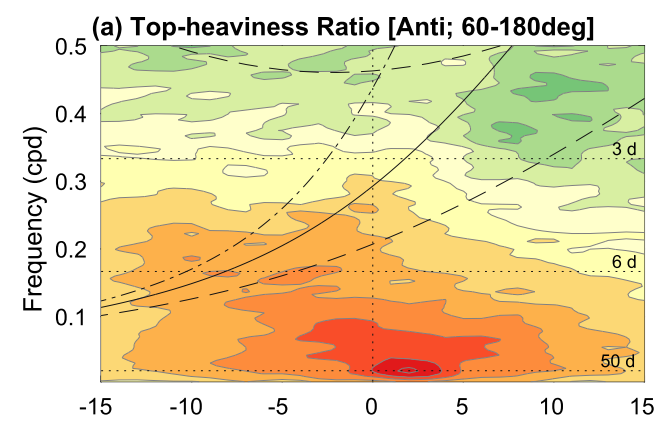

(c) Tilt Ratio [Anti; 60-180deg]

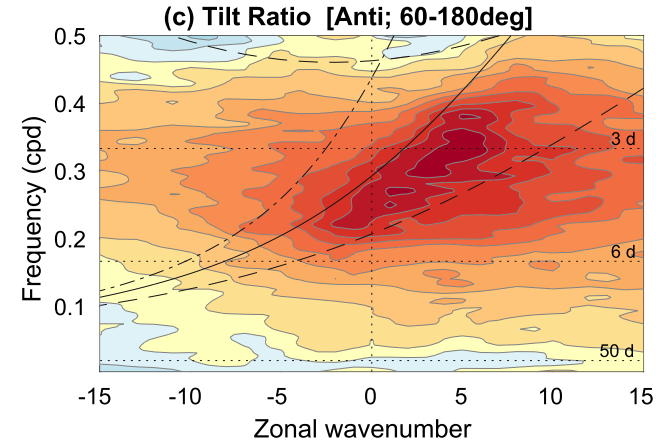

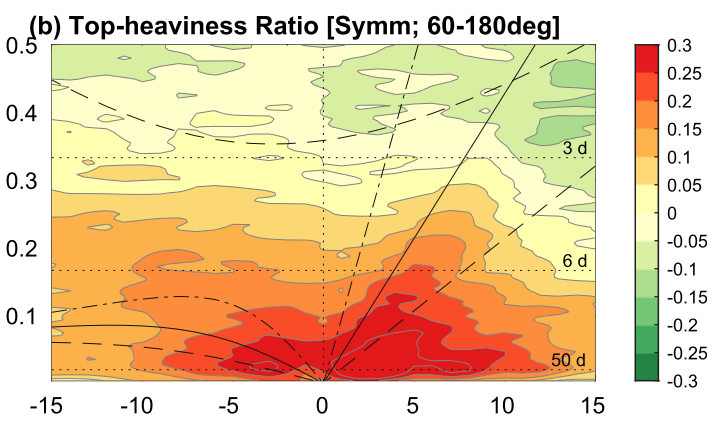

(d) Tilt Ratio [Symm; 60-180deg]

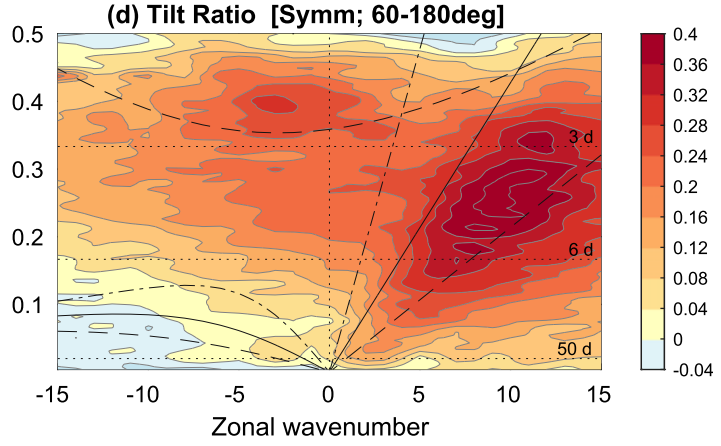

FIG. 9. As in Fig. 5, but with the regional spectra of $o_{1}$ and $o_{2}$ computed within the zonal section of the warm pool region ranging from $60^{\circ} \mathrm{E}$ to $180^{\circ}$.

from Figs. 5 and 7. Therefore, the geographic variability of omega profiles do not affect the main findings in this study.

We also reproduced Figs. 5 and 7 over the Western Hemisphere $\left(180^{\circ}\right.$ to $\left.360^{\circ} \mathrm{E}\right)$. We found that the topheaviness ratios there are significantly smaller in the entire wavenumber-frequency domain than those over the globe, and the tilt ratios exhibit, more or less, similar patterns to the global tilt ratios (not shown). The (effective) GMS figures over the Western Hemisphere exhibit strong signals of the damped Kelvin waves both in the antisymmetric and symmetric components as in Figs. 7a, 7b, 7e, and 7f (not shown). However, those results do not affect the main conclusions of this study, and thus the figures are not shown.

\section{Discussion}

\section{a. Caveats of analyses}

The biggest caveat of this study is the fidelity with which ERA-I captures the CCEW and MJO variability. The ERA-I data are produced based on the Cy31r1 version of the ECMWF Integrated Forecasting System, whose seasonal integrations significantly underestimate the variability of the CCEWs and MJO (e.g., Bechtold et al. 2008). As a result, the ERA-I precipitation has systematic discrepancies in the variance of all the CCEWs and MJO, compared to observations (e.g., Kim et al. 2014b). This deficiency of the simulation skill causes nonnegligible residuals in the MSE budget, which makes MSE budget analyses with ERA-I difficult (e.g., Kim et al. 2014a; Yasunaga et al. 2019). Because the omega profiles are strongly constrained by the simulated convection, it is not surprising the omega profiles in ERA-I contain significant biases.

Nevertheless, it is unclear how much our results are contaminated by the systematic underestimation of the tropical wave variability in the ERA-I dataset. All of our results are computed as a ratio between two quantities, and thus are independent of the amplitude of the wave signals. For instance, the top-heaviness and tilt ratios are a ratio of $\mathrm{PC} 2$ to $\mathrm{PC} 1$. Thus, even if the wave signals are underestimated, as long as the phase relationship between PC1 and PC2 is realistic, the results capture realistic structures of omega profiles in the waves. For more decisive conclusions, we must reproduce all the results with independent datasets such as MERRA-2 (Gelaro et al. 2017), ERA-5 (ECMWF 2017), and if possible, satellite observations, which is left for future work.

Another caveat is an interpretation of our diagnostic method. Our analyses assume the phase relationship between PC1 and PC2 in each wave is locked. But in the reality, each individual wave event might have different structures of omega profiles, and thus different phase 
(a) Vertical GMS [Anti, 60-180deg]

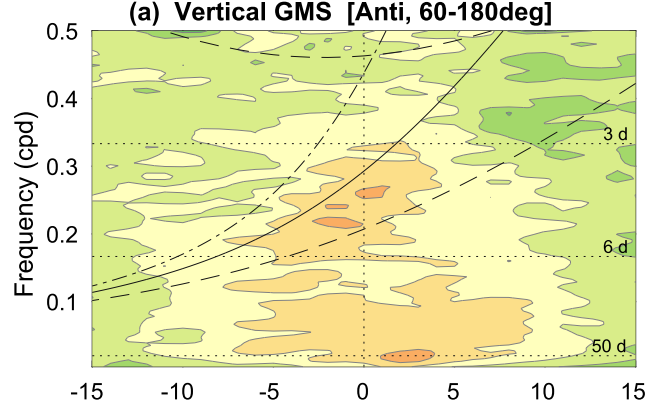

(c) Cld-Rad Feedback [Anti, 60-180deg]

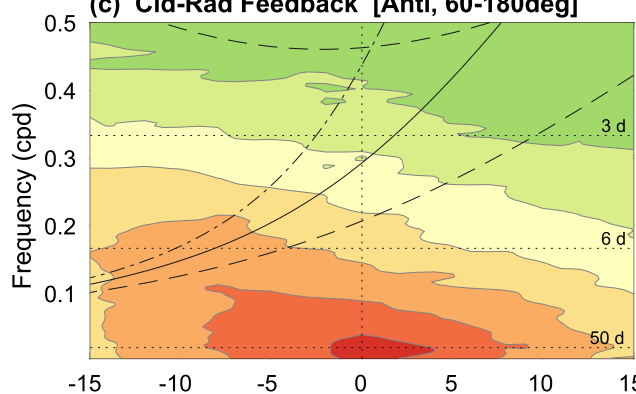

(e) Effective GMS [Anti, 60-180deg]

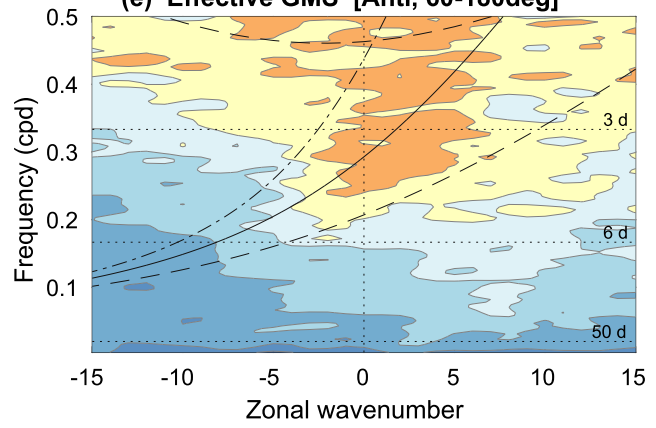

(b) Vertical GMS [Symm, 60-180deg]
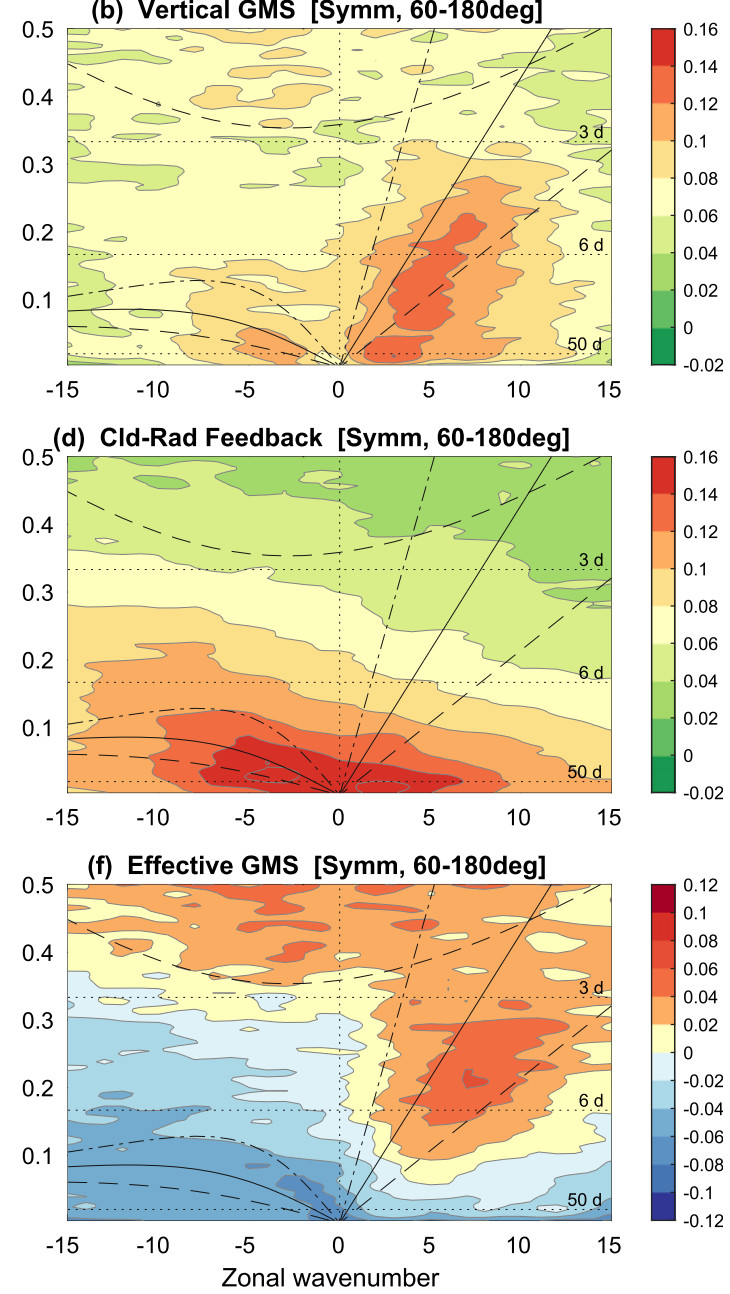

FIG. 10. As in Fig. 7, but with the regional spectra of $\langle\omega \partial h / \partial p\rangle,\langle\omega \partial s / \partial p\rangle$, and $\left\langle Q_{R}\right\rangle$ computed within the zonal section of the warm pool region ranging from $60^{\circ} \mathrm{E}$ to $180^{\circ}$.

relationships. Therefore, we should interpret the results in Figs. 5 and 9 as the most canonical structures of each wave.

Furthermore, it should be noted that our analyses are independent of the amplitude of an event, which may cause differences from traditional composite analyses. In general, structures extracted with composite analyses primarily reflect the characteristics of events with strong amplitudes. In contrast, the top-heaviness and tilt ratios computed in the Fourier space are independent of the amplitude of events, thus each individual event is weighted evenly regardless of its amplitude. For instance, Inoue and Back (2015a) found the structure of the WIG waves to have more top-heavy profiles than those in this study. This may be because most of the strong WIG wave events in their study happen within the envelope of the MJO (see Fig. 1 there), thus the structures of the WIG waves in their study may reflect the background structures of the MJO, which has a topheavy profile.

\section{b. Top-heaviness and the instability of moisture modes}

We have observed that 1) the MJO has the most topheavy omega profile and 2) the GMS on the MJO scale is relatively high, but 3 ) the effective GMS exhibits a negative value due to a strong cloud-radiation feedback. Based on these results, we hypothesize a relationship between omega profile shapes and the instability of the moisture modes, which contrasts with the traditional thinking.

Figure 11 illustrates the relationship between the topheaviness, the GMS, and the cloud-radiation feedback. The red curves in the smaller panels depict typical omega profiles corresponding to a top-heavy system (Fig. 11a) and a bottom-heavy system (Fig. 11b). The background color gradation represents the amount of MSE, and the 
(a) Top-heavy System

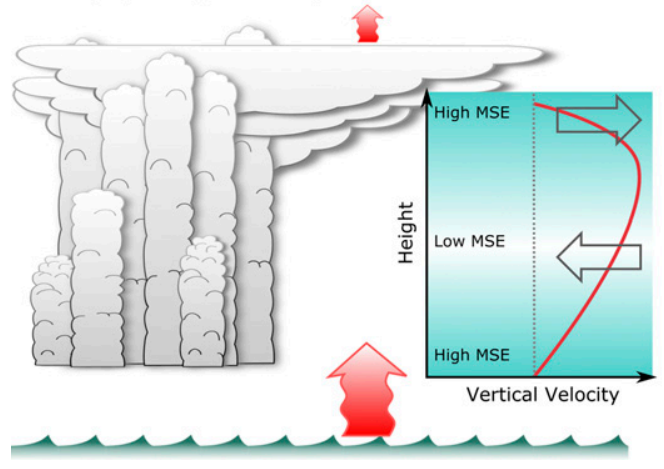

(b) Bottom-heavy System

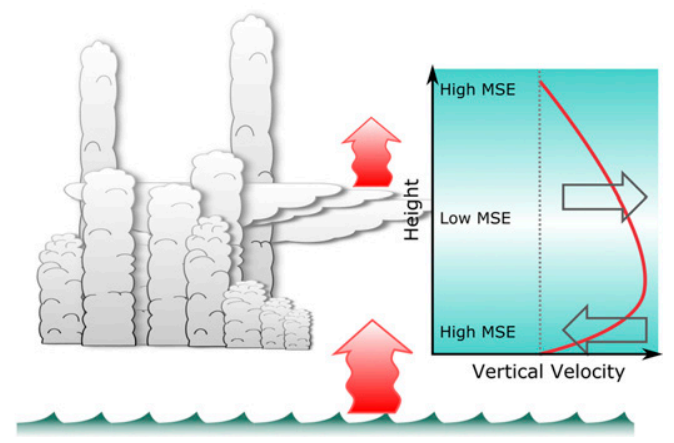

FIG. 11. (a) Schematic of the relationship between the top-heaviness of a convective system, the GMS, and the cloud-radiation feedback. (b) As in (b), but for bottom-heaviness. The wavy red arrows represent the longwave radiative cooling to space. The corresponding omega profiles (red curves) with their associated large-scale circulations (gray arrows) are also depicted. The left-pointing (right-pointing) arrows represent the convergence (divergence) of the air. The background color gradation represents the amount of MSE. The top-heavy omega profile in (a) is associated with high GMS, while the bottom-heavy profile in (b) is associated with low GMS.

nonfilled arrows represent the large-scale circulations. The top-heavy system imports the low-MSE air in the midtroposphere, and exports the high-MSE air in the upper troposphere, leading to a high value of the GMS. In contrast, the bottom-heavy system imports the highMSE air in the lower troposphere, and exports the lowMSE air in the midtroposphere, leading to a low value of the GMS. Therefore, from the perspective of the GMS, the bottom-heavy system is more favorable for the MSE buildup, and thus for the moisture modes.

On the other hand, the cloud-radiation feedback competes with the damping effect due to the GMS, as pointed out by many previous studies (e.g., Lin and Mapes 2004; Maloney 2009; Andersen and Kuang 2012; Chikira 2014; Wolding and Maloney 2015; Sobel et al. 2014; Janiga and Zhang 2016; Wolding et al. 2016). The top-heavy system, which is associated with a high fraction of stratiform clouds (e.g., Schumacher et al. 2004), may have a strong greenhouse effect that reduces the radiative cooling into the space. Such a radiative heating anomaly effectively acts as a source of MSE. On the other hand, the bottom-heavy system with a small amount of stratiform clouds may not have such a strong greenhouse effect, thus allowing the radiation to escape into the space. Thus, from the perspective of the cloudradiation feedback, the top-heavy system may be more favorable for the growth of the moisture modes.

Therefore, two opposing effects may be competing with each other in the bottom-heavy and the top-heavy systems. In the bottom-heavy system, the damping effect due to the GMS is small, but the amplification effect due to the cloud-radiation feedback is also small. In contrast, in the top-heavy system, the damping effect due to the GMS is large, but the amplification effect due to the cloud-radiation feedback may be larger than the damping effect of the GMS. As a result, it may be the top-heavy system-rather than the bottom-heavy system-that is more favorable (or more unstable) for the moisture modes, which contrasts with the traditional view that a bottom-heavy system with low GMS is more favorable for the moisture modes.

This idea could be supported by previous GCM studies. For instance, Fu and Wang (2009) found that a significant fraction of stratiform convection, which is associated with a top-heavy omega profile, is necessary to simulate a robust MJO in an atmospheric GCM. Seo and Wang (2010) also claimed that the stratiform convection is one of the most important factors in good MJO simulations.

It is worth noting that, while elevated stratiform clouds result in stronger anomalous radiative heating, this elevated heating feeds back into the vertical velocity. Longwave radiative heating in stratiform clouds exhibits a top-heavy shape, and in the weak temperature gradient balance (Sobel et al. 2001) this top-heavy heating induces top-heavy vertical velocities (e.g., Wolding et al. 2016). Thus, there may exist a positive feedback loop between the top-heaviness and the enhanced longwave radiative heating, which maintains a preferable condition for the moisture modes. Since our results are purely diagnostic, we cannot say how much of the top-heaviness in the MJO is a result of the radiative heating itself, though we can infer that, for initiating this positive feedback loop, elevated stratiform clouds or topheavy omega profiles are necessary.

\section{c. Interpretation of tilts in CC gravity waves}

The tilted structures in the CC gravity waves may be interpreted as a manifestation of the coupling between 
convection and convective suppression mechanisms. The convective suppression mechanisms may include convective inhibition (CIN) (e.g., Raymond and Fuchs 2007) (RF07) and a midtropospheric saturation deficit (e.g., Kuang 2008b). This coupling regulates the transition from shallow to deep convection, making the relationship between a warm anomaly and positive heating in-phase (see Fig. 11 in Kuang 2008b). This inphase relationship-warm gets warmer and cold gets colder-causes the instability of the CC gravity waves. Without the tilted structure, a warm anomaly becomes out-of-phase with positive heating, which damps a temperature anomaly.

The importance of the tilt can be understood by comparing two theoretical studies: Fuchs and Raymond (2007) (FR07) and RF07. These two theories are identical except for the convective parameterizations; Only in RF07, the CIN dependency of the convection is implemented [Eq. (7) therein]. As a result, RF07 produces an unstable CC gravity wave mode that has a tilted structure whereas the CC gravity wave mode in FR07 is vertically stacked and damped. Therefore, from this comparison, we can infer that the tilted structures in the CC gravity waves are created by the coupling between the convection and the convective suppression due to $\mathrm{CIN}$, and this coupling is crucial for the destabilization of the $\mathrm{CC}$ gravity wave mode.

\section{Summary}

By utilizing the ERA-I datasets, we investigated the structures of the omega profiles in the tropical wave disturbances including the Kelvin, equatorial Rossby (ER), westward inertio-gravity (WIG), eastward inertiogravity (EIG), mixed Rossby-gravity (MRG), tropical depression (TD)-type waves, and the MJO. For quantitatively assessing the structures of the omega profiles for each wave, we defined two quantities: the topheaviness ratio and the tilt ratio. The top-heaviness ratio represents how top-heavy (or bottom-heavy) an omega profile is at the convective maximum; and the tilt ratio represents how much tilt omega profiles in a wave contain in the spatiotemporal evolution.

The top-heaviness ratio and the tilt ratio are defined with the first two leading EOF-PC pairs; the top-heaviness ratio and the tilt ratio are defined, respectively, as the cospectrum and as the quadrature spectrum of the $\mathrm{PC} 1$ and $\mathrm{PC} 2$, divided by the power spectrum of PC1. Because the first two leading EOF-PC pairs explain most of the spatiotemporal variance of the omega profiles among all the waves (at least $75 \%$ for all the waves, and greater than $90 \%$ for the MJO), the values of the top-heaviness and tilt ratios succinctly summarize the important properties of the omega profiles in the tropical waves.

By using these quantities, we have answered the following questions. 1) Which waves have a more topheavy or more bottom-heavy omega profile shape? 2) Which wave has tilted (or vertically stacked) omega profiles in its spatiotemporal evolution? We found that, in general, omega profiles become more top-heavy as the time scale gets longer and the spatial scale gets larger; and thus, the MJO has the most top-heavy omega profile, while the WIG and EIG waves have the most bottom-heavy profile among all the waves. The tilt ratio has a distinct pattern in the wavenumber-frequency domain. The Kelvin, WIG, EIG, and MRG waves, which are hypothetically categorized as the fast-moving convectively coupled (CC) gravity wave modes, have significant tilts of the omega profiles in their spatiotemporal evolution, while the ER wave and MJO, which are associated with the slow-moving moisture modes, have more vertically stacked profiles than the other waves. These results of the tilts strongly support the theory by Raymond and Fuchs (2007), who proposed a simple model which elucidates the mechanisms of the $\mathrm{CC}$ gravity wave mode and the moisture mode as separate eigenmodes.

We also computed the (vertical) gross moist stability (GMS), cloud-radiation feedback, and effective GMS in the wavenumber-frequency domain in the same way as the computation of the top-heaviness ratio. The patterns of the GMS in the wavenumber-frequency domain are largely consistent with those of the top-heaviness ratio-although there are some differences in the details. The MJO with the most top-heavy omega profile has a high value of the GMS, which is believed to be unfavorable for the growth of the moisture modes. However, the effective GMS (the GMS minus the cloud-radiation feedback) exhibits a negative value on the MJO scale, which is favorable for the moisture mode instability. This result is due to a strong cloudradiation feedback, which surpasses the high value of the GMS. Interestingly, the MJO, ER, and TD-type waves, which are associated with the slow-moving moisture modes, have negative values of the effective GMS, while the Kelvin, WIG, EIG, and MRG waves, categorized as the fast-moving CC gravity wave modes, have positive values of the effective GMS, especially over the warm pool region. That may be linked to different mechanisms of the CC gravity wave modes and the moisture modes.

Our results may reject the hypothesis proposed by Kuang (2011), who claimed that the MJO with the longest zonal wavelength has the most bottom-heavy omega profile and thus a low value of the GMS that may be responsible for the scale selection of the MJO. 
Our analyses show the opposite results; the MJO has the most top-heavy profile among all the waves, and thus a high value of the GMS.

Based on our analyses, we speculated a relationship between omega profile shapes and the instability of the moisture modes. In a bottom-heavy system, the damping effect due to the GMS is small, but the amplification effect due to the cloud-radiation feedback is also small. In contrast, in a top-heavy system, the damping effect due to the GMS is large, but the amplification effect due to the cloud-radiation feedback is also large. We hypothesized that the effect of the cloud-radiation feedback generally surpasses the effect of the GMS, and as a result, a top-heavy omega profile with a stronger cloudradiation feedback due to stratiform clouds becomes more favorable for the growth of the moisture modes. This contrasts with the traditional thinking that a bottom-heavy omega profile with low GMS is more favorable for the moisture modes.

The analyses of the top-heaviness ratio and the tilt ratio can succinctly summarize the important properties of the omega profiles in different waves. Therefore, the analyses proposed here provide a useful diagnostic framework for the assessment of the tropical wave disturbances in general circulation models, especially for climate model intercomparison projects.

Acknowledgments. We are grateful for valuable comments by Brandon Wolding and two anonymous reviewers. K. Inoue was supported by the NOAA Climate Program Office under Award NA15OAR4310177 and by an appointment to the NASA Postdoctoral Program at the NASA Goddard Institute for Space Studies, administered by Universities Space Research Association. Á. F. Adames was supported by the National Science Foundation's Grant AGS-1841559. K. Yasunaga was supported by JSPS KAKENHI Grants JP25400463 and JP16KK0095.

\section{APPENDIX}

\section{Estimation of Background Power Spectra}

In this appendix, we discuss how the background power spectra were estimated in this study, especially for the OLR in ERA-I. The procedure is similar to the one used in Wheeler and Kiladis (1999), with some modifications discussed below.

The procedure in Wheeler and Kiladis (1999) consists of four steps:

1) add the raw power spectra of the antisymmetric and symmetric components together;
2) apply a 1-2-1 running-mean filter 10 times in the temporal-frequency dimension (along a constant zonal wavenumber);

3 ) in the zonal wavenumber dimension (along a constant temporal frequency), apply the 1-2-1 filter as well, but the number of the pass of the filter is determined based on the temporal frequencies, being 10 at low frequencies and increased to 40 at higher frequencies; and

4) divide the computed background power spectra by 2 for applying to the antisymmetric and symmetric components.

We followed steps 1 and 2 in the same way. In step 3, Wheeler and Kiladis (1999) increase the number of the filter passes from 10 to 40 stepwise with two different steps (like 10 to 20 to 40, etc.) In this study, we determined this number with the round function of $\exp (3.7328$ freq +2.2279$)$, which yields 10 at $0.02 \mathrm{cpd}$ (or 50 -day periodicity) and 60 at $0.5 \mathrm{cpd}$ (or 2-day periodicity), increasing exponentially as a function of temporal frequencies (freq). We introduced this function simply because the details of the stepwise increase are not specified in Wheeler and Kiladis (1999), and the results are not sensitive to the choice of how to increase the filtering number.

We found that step 4 requires some modification for applying to the ERA-I data. In common applications, we divide the computed background power spectra by 2 for applying to the antisymmetric and symmetric components. This is based on an implicit assumption that the total background variance of the antisymmetric and symmetric components is the same so that the power can be evenly split. However, this assumption does not work for the ERA-I data.

Figure A1 is a normalized space-time spectral diagram-including the data over land-whose background power spectra were simply divided by 2 . One might interpret this figure as an underestimation of the MRG and EIG waves, and an overestimation of the TD-type wave in the ECMWF model, compared to the observation. One might also think that the power spectrum in the symmetric component of the ERA-I data are much noisier than that of the observational data. However, we will show that this result is very sensitive to the computational method of background power spectra, and thus we might need a "tailored method" for the computation of background power spectra for different datasets.

For showing this, we first compared the total spatiotemporal variance in the antisymmetric and symmetric components for both the observation and ERA-I. From Parseval's theorem, the total spatiotemporal variance of 
TABLE A1. Total variance and NW variance defined in Eq. (A2) of OLR in the observation and ERA-I for the symmetric and antisymmetric components and the ratios between them.

\begin{tabular}{lccccc}
\hline \hline & \multicolumn{2}{c}{ Observed OLR } & & \multicolumn{2}{c}{ ERA-I OLR } \\
\cline { 2 - 3 } & Total variance & NW variance & & Total variance & NW variance \\
\hline Symmetric $\left(\mathrm{W} \mathrm{m}^{-2}\right)^{2}$ & 441.63 & 29.13 & & 276.28 & 7.83 \\
Antisymmetric $\left(\mathrm{W} \mathrm{m}^{-2}\right)^{2}$ & 371.05 & 28.82 & & 199.14 & 6.84 \\
Ratio & 1.19 & 1.01 & & 1.39 & 1.15 \\
\hline
\end{tabular}

$a\left(x_{m}, t_{n}\right)$ is computed with the summation of the Fourier coefficients, as

$$
\frac{1}{M N} \sum_{m=1}^{M} \sum_{n=1}^{N} a^{2}\left(x_{m}, t_{n}\right)=\sum_{k=1}^{M} \sum_{l=1}^{N}|A(k, l)|^{2}
$$

Table A1 summarizes the total spatiotemporal variance of the OLR computed with Eq. (A1) for the antisymmetric and symmetric components in the observation and ERA-I.

In the observational OLR, the total variance of the symmetric and antisymmetric component is 441.63 and $371.05\left(\mathrm{~W} \mathrm{~m}^{-2}\right)^{2}$, respectively; and the ratio between them is 1.19 . This ratio means that the total variance of the symmetric component in the observational OLR is about $20 \%$ greater than that of the antisymmetric component. In contrast, the total variance of the symmetric and antisymmetric component in the ERA-I's OLR is 276.28 and $199.14\left(\mathrm{~W} \mathrm{~m}^{-2}\right)^{2}$, respectively. The ratio between them is 1.39 , which indicates that the total variance of the symmetric component is about $40 \%$ greater than that of the antisymmetric component in ERA-I. In summary, in both the observation and ERA-I, there exists an imbalance of the total variance between the antisymmetric and the symmetric components; and the imbalance in ERA-I is much greater than that in the observation. Therefore, when computing the background power spectra for the antisymmetric and the symmetric components, that imbalance must be taken into account.

We define the "background adjustment ratios" for the antisymmetric and symmetric components, which adjust the imbalance of the variance between the two components. For computing them, however, the total variance discussed above should not be used because it contains the variance due to the wave signals. In general, the variance of the symmetric component may be greater than that of the antisymmetric component because the

(a) Obs. OLR [Anti; Global; No Adj.]
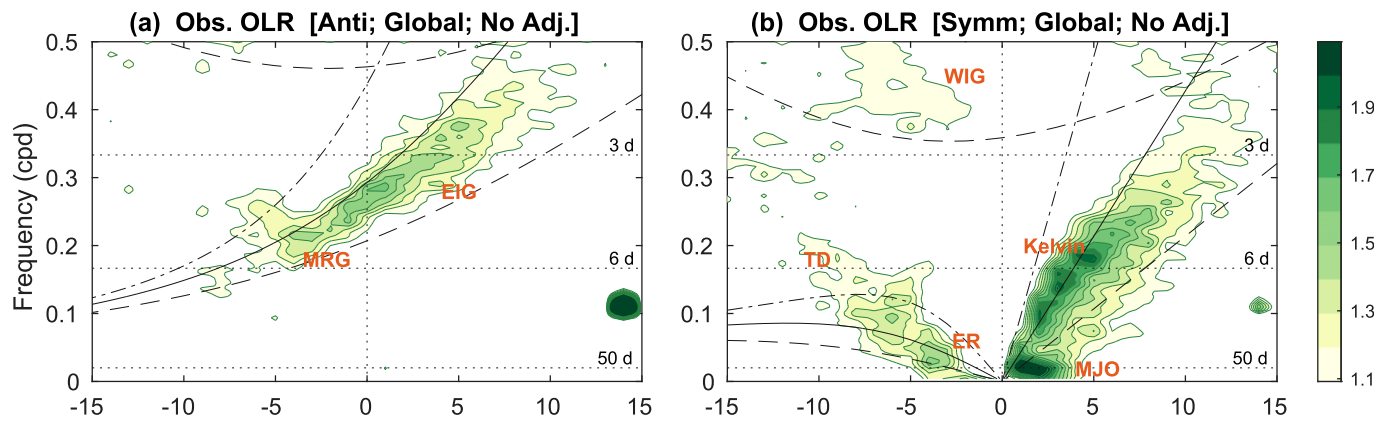

(c) ERAI OLR [Anti; Global; No Adj.]
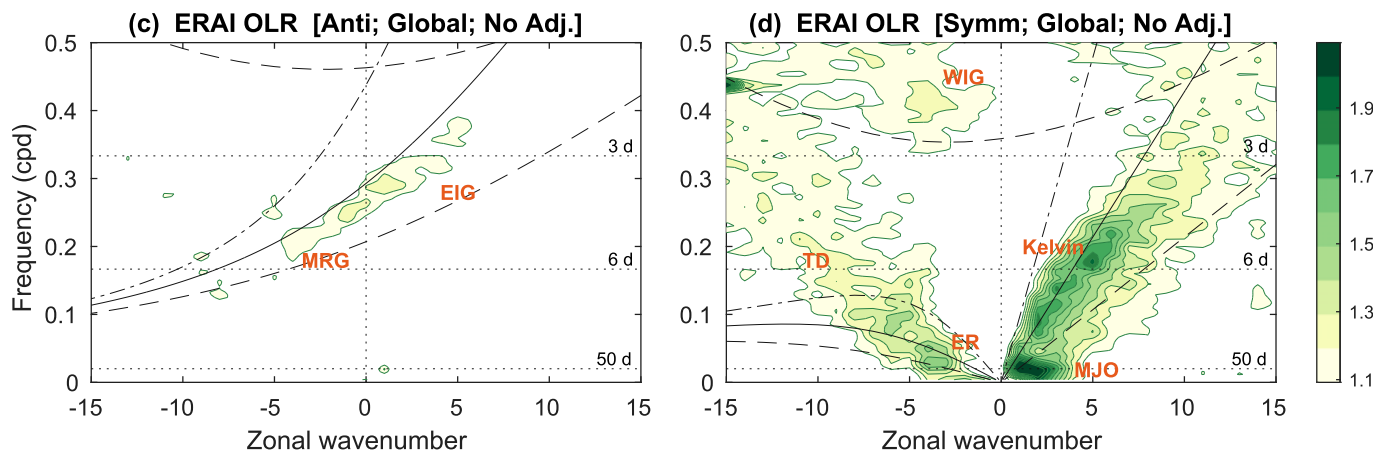

FIG. A1. As in Fig. 3, but the background power spectra for the (a),(c) antisymmetric and (b),(d) symmetric components are not adjusted (simply divided by 2 ), and the land data are not masked out. 
(a) Obs. OLR [Anti; Global; Adj. B.Noise]

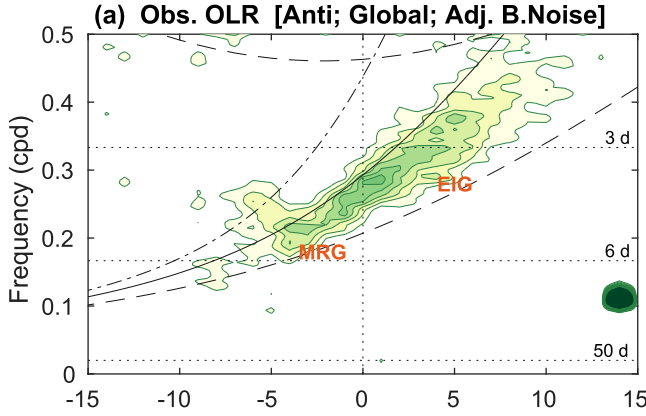

(c) ERAI OLR [Anti; Global; Adj. B.Noise]

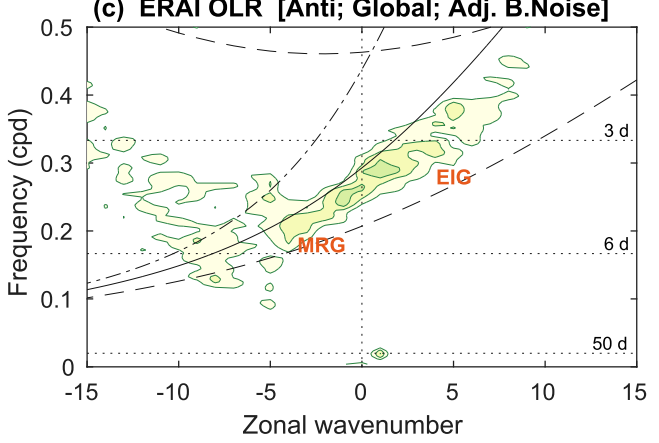

(b) Obs. OLR [Symm; Global; Adj. B.Noise]

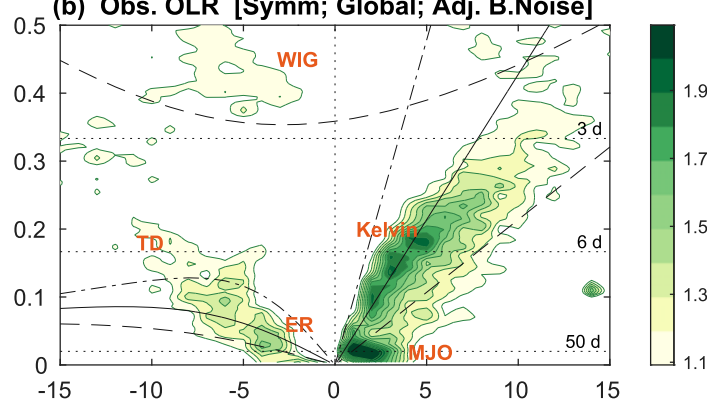

(d) ERAI OLR [Symm; Global; Adj. B.Noise]

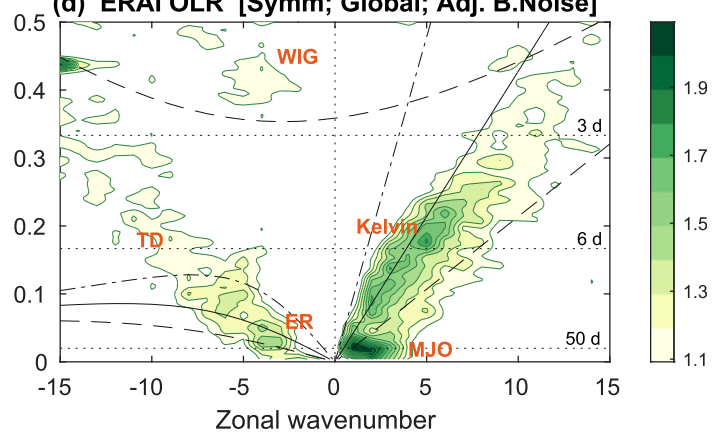

FIG. A2. As in Fig. A1, but the background spectra are adjusted with the background adjustment ratios defined in Eq. (A3).

MJO in the symmetric component contributes to a significant amount of the variance. We need to adjust the background power spectra based on the imbalance of the background nonwave variance.

For that reason, we define the nonwave (NW) variance as

$$
\Lambda=\sum_{k, l \in \text { nonwave }}|A(k, l)|^{2}
$$

which represents the summation of the power spectra over the "nonwave spectral region," $k, l, \in$ nonwave, defined as the spectral region of $|k|>25$ and the temporal frequencies greater than $1 / 20 \mathrm{cpd}$. This choice is based on the assumption that small-scale, high-frequency variability is almost purely due to the background nonwave variance. And we define the antisymmetric and symmetric background adjustment ratios $\alpha_{a}$ and $\alpha_{s}$ as

$$
\alpha_{a}=\frac{\Lambda_{a}}{\Lambda_{a}+\Lambda_{s}}, \quad \alpha_{s}=\frac{\Lambda_{s}}{\Lambda_{a}+\Lambda_{s}},
$$

where $\Lambda_{a}$ and $\Lambda_{s}$ are the NW variance of the antisymmetric and symmetric components, respectively. Then, the background power spectra for the antisymmetric and symmetric components are computed by multiplying the total background power spectra with the adjustment ratio for each component, instead of dividing them by 2 . If the NW variance of the antisymmetric and symmetric components is the same, the adjustment ratio becomes 0.5 , which is equivalent to the division by 2 .

The NW variance of the OLR in the observation and ERA-I is summarized in Table A1. The symmetric and antisymmetric NW variance in the observation is 29.13 and $28.82\left(\mathrm{~W} \mathrm{~m}^{-2}\right)^{2}$, respectively; and the ratio between them is 1.01 , which is close to unity. As a result, the antisymmetric and symmetric background adjustment ratios are 0.4973 and 0.5027 , respectively. This is why the traditional procedure in step 4 , the division of the total background power by 2 , has not been an issue for the observational data. The imbalance of the NW variance between the antisymmetric and symmetric components is so small that we can evenly split the background power for each component. Figures A2a and A2b are normalized spectral diagrams of the observational OLR whose background power spectra were adjusted with the background adjustment ratios. Those are almost identical to Figs. A1a and A1b with no adjustment; this result indicates that the observational OLR does not require the background adjustment.

In contrast, the symmetric and antisymmetric NW variance of the OLR in ERA-I is 7.83 and $6.84\left(\mathrm{~W} \mathrm{~m}^{-2}\right)^{2}$, respectively; the symmetric NW variance is $15 \%$ greater than that of the antisymmetric component. This leads to the antisymmetric and symmetric background adjustment ratios being 0.4663 and 0.5337 , 
respectively. Figures A2c and A2d are normalized spectral diagrams of the ERA-I's OLR with the background power adjusted with $\alpha_{a}$ and $\alpha_{s}$. Compared to Figs. A1c and A1d, the signals of the MRG and EIG waves are much more significant, and the symmetric signals are less noisy. In this figure, the spectral signals of ERA-I are largely comparable to those of the observational data, indicating that the ECMWF model simulates wave signals. The striking difference between Figs. A1 and A2 for ERA-I indicates that a normalized spectral diagram is sensitive to the computed background spectrum, and that careful treatment and interpretation is needed, especially for nonobservational data.

This result may also indicate the caveat of the usage of normalized spectral diagrams for model intercomparison projects. Climate-model intercomparisons have been done for examining GCM's simulation skills of the CCEWs and the MJO (e.g., Lin et al. 2006; Straub et al. 2010; Kim et al. 2009; Benedict et al. 2013; Hung et al. 2013; Jiang et al. 2015). However, to the best of our knowledge, no past model intercomparison studies paid attention to the imbalance of the antisymmetric and the symmetric background variance. If each model has a different amount of the antisymmetric-symmetric imbalance of the background variance, as we found in the observation and ERA-I, one can underestimate or overestimate wave signals in a normalized spectral diagram as demonstrated in Figs. A1 and A2.

It should be emphasized that there is no perfect way to compute background power spectra because all the methods used currently-including our adjustment method discussed here-rely on subjective choices. Therefore, when using a normalized spectral diagram, that fact should be kept in mind. Perhaps, a method which is independent of background power spectra, such as a coherence analysis, may be a better choice for investigating the CCEWs and MJO.

\section{REFERENCES}

Adames, Á. F., and J. M. Wallace, 2014: Three-dimensional structure and evolution of the vertical velocity and divergence fields in the MJO. J. Atmos. Sci., 71, 4661-4681, https://doi.org/ 10.1175/JAS-D-14-0091.1.

— , and D. Kim, 2016: The MJO as a dispersive, convectively coupled moisture wave: Theory and observations. J. Atmos. Sci., 73, 913-941, https://doi.org/10.1175/JAS-D-15-0170.1.

_- and Y. Ming, 2018: Interactions between water vapor and potential vorticity in synoptic-scale monsoonal disturbances: Moisture vortex instability. J. Atmos. Sci., 75, 2083-2106, https://doi.org/10.1175/JAS-D-17-0310.1.

—, D. Kim, S. K. Clark, Y. Ming, and K. Inoue, 2019: Scale analysis of moist thermodynamics in a simple model and the relationship between moisture modes and gravity waves.
J. Atmos. Sci., 76, 3863-3881, https://doi.org/10.1175/JAS-D19-0121.1.

Andersen, J. A., and Z. Kuang, 2012: Moist static energy budget of MJO-like disturbances in the atmosphere of a zonally symmetric aquaplanet. J. Climate, 25, 2782-2804, https://doi.org/ 10.1175/JCLI-D-11-00168.1.

Back, L. E., and C. S. Bretherton, 2006: Geographic variability in the export of moist static energy and vertical motion profiles in the tropical Pacific. Geophys. Res. Lett., 33, L17810, https:// doi.org/10.1029/2006GL026672.

- , and - 2009: A simple model of climatological rainfall and vertical motion patterns over the tropical oceans. J. Climate, 22, 6477-6497, https://doi.org/10.1175/2009JCLI2393.1.

_ Z Z. Hansen, and Z. Handlos, 2017: Estimating vertical motion profile top-heaviness: Reanalysis compared to satellite-based observations and stratiform rain fraction. J. Atmos. Sci., 74 855-864, https://doi.org/10.1175/JAS-D-16-0062.1.

Bechtold, P., M. Köhler, T. Jung, F. Doblas-Reyes, M. Leutbecher, M. J. Rodwell, F. Vitart, and G. Balsamo, 2008: Advances in simulating atmospheric variability with the ECMWF model: From synoptic to decadal time-scales. Quart. J. Roy. Meteor. Soc., 134, 1337-1351, https://doi.org/10.1002/qj.289.

Benedict, J. J., E. D. Maloney, A. H. Sobel, D. M. Frierson, and L. J. Donner, 2013: Tropical intraseasonal variability in version 3 of the GFDL atmosphere model. J. Climate, 26, 426449, https://doi.org/10.1175/JCLI-D-12-00103.1.

Bretherton, C. S., and A. H. Sobel, 2002: A simple model of a convectively coupled Walker circulation using the weak temperature gradient approximation. J. Climate, 15, 2907-2920, https://doi.org/10.1175/1520-0442(2002)015<2907:ASMOAC > 2.0.CO;2.

Chikira, M., 2014: Eastward-propagating intraseasonal oscillation represented by Chikira-Sugiyama cumulus parameterization. Part II: Understanding moisture variation under weak temperature gradient balance. J. Atmos. Sci., 71, 615-639, https:// doi.org/10.1175/JAS-D-13-038.1.

Dee, D. P., and Coauthors, 2011: The ERA-Interim reanalysis: Configuration and performance of the data assimilation system. Quart. J. Roy. Meteor. Soc., 137, 553-597, https://doi.org/ 10.1002/qj.828.

Dias, J., S. Leroux, S. N. Tulich, and G. N. Kiladis, 2013: How systematic is organized tropical convection within the MJO? Geophys. Res. Lett., 40, 1420-1425, https://doi.org/10.1002/ grl.50308.

ECMWF, 2017: ERA5 reanalysis. European Centre for MediumRange Weather Forecasts, accessed 23 March 2020, https:// www.ecmwf.int/en/forecasts/datasets/reanalysis-datasets/era5.

Emanuel, K. A., J. D. Neelin, and C. S. Bretherton, 1994: On large-scale circulations in convecting atmospheres. Quart. J. Roy. Meteor. Soc., 120, 1111-1143, https://doi.org/ 10.1002/qj.49712051902.

Fu, X., and B. Wang, 2009: Critical roles of the stratiform rainfall in sustaining the Madden-Julian oscillation: GCM experiments. J. Climate, 22, 3939-3959, https://doi.org/10.1175/ 2009JCLI2610.1.

Fuchs, Ž., and D. J. Raymond, 2007: A simple, vertically resolved model of tropical disturbances with a humidity closure. Tellus, 59A, 344-354, https://doi.org/10.1111/j.1600-0870.2007.00230.x. — J. Adv. Model. Earth Syst., 9, 1195-1211, https://doi.org/ 10.1002/2017MS000963.

_ S. Gjorgjievska, and D. J. Raymond, 2012: Effects of varying the shape of the convective heating profile on convectively 
coupled gravity waves and moisture modes. J. Atmos. Sci., 69 , 2505-2519, https://doi.org/10.1175/JAS-D-11-0308.1.

— D. J. Raymond, and S. Sentić, 2019: A simple model of convectively coupled equatorial Rossby waves. J. Adv. Model. Earth Syst., 11, 173-184, https://doi.org/10.1029/2018MS001433.

Gelaro, R., and Coauthors, 2017: The Modern-Era Retrospective Analysis for Research and Applications, version 2 (MERRA2). J. Climate, 30, 5419-5454, https://doi.org/10.1175/JCLI-D16-0758.1.

Haertel, P. T., and G. N. Kiladis, 2004: Dynamics of 2-day equatorial waves. J. Atmos. Sci., 61, 2707-2721, https://doi.org/10.1175/ JAS3352.1.

,,-- A. Denno, and T. M. Rickenbach, 2008: Vertical-mode decompositions of 2-day waves and the Madden-Julian oscillation. J. Atmos. Sci., 65, 813-833, https://doi.org/10.1175/ 2007JAS2314.1.

Hendon, H. H., and M. C. Wheeler, 2008: Some space-time spectral analyses of tropical convection and planetary-scale waves. J. Atmos. Sci., 65, 2936-2948, https://doi.org/10.1175/ 2008JAS2675.1.

Herman, M. J., Ž. Fuchs, D. J. Raymond, and P. Bechtold, 2016: Convectively coupled Kelvin waves: From linear theory to global models. J. Atmos. Sci., 73, 407-428, https://doi.org/ 10.1175/JAS-D-15-0153.1.

Hung, M.-P., J.-L. Lin, W. Wang, D. Kim, T. Shinoda, and S. J. Weaver, 2013: MJO and convectively coupled equatorial waves simulated by CMIP5 climate models. J. Climate, 26, 6185-6214, https://doi.org/10.1175/JCLI-D-12-00541.1.

Inoue, K., and L. Back, 2015a: Column-integrated moist static energy budget analysis on various time scales during TOGA COARE. J. Atmos. Sci., 72, 1856-1871, https://doi.org/10.1175/ JAS-D-14-0249.1.

$\longrightarrow$, and,$- 2015 \mathrm{~b}$ : Gross moist stability assessment during TOGA COARE: Various interpretations of gross moist stability. J. Atmos. Sci., 72, 4148-4166, https://doi.org/10.1175/ JAS-D-15-0092.1.

Janiga, M. A., and C. Zhang, 2016: MJO moisture budget during DYNAMO in a cloud-resolving model. J. Atmos. Sci., 73, 2257-2278, https://doi.org/10.1175/JAS-D-14-0379.1.

Jiang, X., and Coauthors, 2015: Vertical structure and physical processes of the Madden-Julian oscillation: Exploring key model physics in climate simulations. J. Geophys. Res. Atmos., 120, 4718-4748, https://doi.org/10.1002/2014JD022375.

Khouider, B., and A. J. Majda, 2006: A simple multicloud parameterization for convectively coupled tropical waves. Part I: Linear analysis. J. Atmos. Sci., 63, 1308-1323, https://doi.org/ 10.1175/JAS3677.1.

Kiladis, G. N., K. H. Straub, and P. T. Haertel, 2005: Zonal and vertical structure of the Madden-Julian oscillation. J. Atmos. Sci., 62, 2790-2809, https://doi.org/10.1175/JAS3520.1.

- M. C. Wheeler, P. T. Haertel, K. H. Straub, and P. E. Roundy, 2009: Convectively coupled equatorial waves. Rev. Geophys., 47, RG2003, https://doi.org/10.1029/2008RG000266.

Kim, D., and Coauthors, 2009: Application of MJO simulation diagnostics to climate models. J. Climate, 22, 6413-6436, https://doi.org/10.1175/2009JCLI3063.1.

_, J.-S. Kug, and A. H. Sobel, 2014a: Propagating versus nonpropagating Madden-Julian oscillation events. J. Climate, 27, 111-125, https://doi.org/10.1175/JCLI-D-13-00084.1.

, M.-I. Lee, D. Kim, S. D. Schubert, D. E. Waliser, and B. Tian, 2014b: Representation of tropical subseasonal variability of precipitation in global reanalyses. Climate Dyn., 43, 517-534, https://doi.org/10.1007/s00382-013-1890-x.
— M.-S. Ahn, I.-S. Kang, and A. D. Del Genio, 2015: Role of longwave cloud-radiation feedback in the simulation of the Madden-Julian oscillation. J. Climate, 28, 6979-6994, https:// doi.org/10.1175/JCLI-D-14-00767.1.

Kuang, Z., 2008a: Modeling the interaction between cumulus convection and linear gravity waves using a limited-domain cloud system-resolving model. J. Atmos. Sci., 65, 576-591, https://doi.org/10.1175/2007JAS2399.1.

- 2008b: A moisture-stratiform instability for convectively coupled waves. J. Atmos. Sci., 65, 834-854, https://doi.org/ 10.1175/2007JAS2444.1.

_ 2011: The wavelength dependence of the gross moist stability and the scale selection in the instability of column-integrated moist static energy. J. Atmos. Sci., 68, 61-74, https://doi.org/ 10.1175/2010JAS3591.1.

Liebmann, B., and H. H. Hendon, 1990: Synoptic-scale disturbances near the equator. J. Atmos. Sci., 47, 1463-1479, https://doi.org/ 10.1175/1520-0469(1990)047<1463:SSDNTE > 2.0.CO;2.

— lated) outgoing longwave radiation dataset. Bull. Amer. Meteor. Soc., 77, 1275-1277.

Lin, J.-L., and B. E. Mapes, 2004: Radiation budget of the tropical intraseasonal oscillation. J. Atmos. Sci., 61, 2050-2062, https:// doi.org/10.1175/1520-0469(2004)061<2050:RBOTTI>2.0.CO;2. _, _- M. Zhang, and M. Newman, 2004: Stratiform precipitation, vertical heating profiles, and the Madden-Julian Oscillation. J. Atmos. Sci., 61, 296-309, https://doi.org/10.1175/ 1520-0469(2004)061<0296:SPVHPA>2.0.CO;2.

—, and Coauthors, 2006: Tropical intraseasonal variability in 14 IPCC AR4 climate models. Part I: Convective signals. J. Climate, 19, 2665-2690, https://doi.org/10.1175/JCLI3735.1.

Madden, R. A., and P. R. Julian, 1971: Detection of a 40-50 day oscillation in the zonal wind in the tropical Pacific. J. Atmos. Sci., 28, 702-708, https://doi.org/10.1175/1520-0469(1971) 028<0702:DOADOI $>2.0 . \mathrm{CO} ; 2$.

- - and ——, 1972: Description of global-scale circulation cells in the tropics with a 40-50 day period. J. Atmos. Sci., 29, 1109-1123, https://doi.org/10.1175/1520-0469(1972) 029<1109:DOGSCC $>2.0$. CO 2 .

Maloney, E. D., 2009: The moist static energy budget of a composite tropical intraseasonal oscillation in a climate model. J. Climate, 22, 711-729, https://doi.org/10.1175/2008JCLI2542.1.

Mapes, B. E., 2000: Convective inhibition, subgrid-scale triggering energy, and stratiform instability in a toy tropical wave model. J. Atmos. Sci., 57, 1515-1535, https://doi.org/10.1175/15200469(2000)057<1515:CISSTE > 2.0.CO;2.

- S. Tulich, J. Lin, and P. Zuidema, 2006: The mesoscale convection life cycle: Building block or prototype for large-scale tropical waves? Dyn. Atmos. Oceans, 42, 3-29, https://doi.org/ 10.1016/j.dynatmoce.2006.03.003.

Matsuno, T., 1966: Quasi-geostrophic motions in the equatorial area. J. Meteor. Soc. Japan, 44, 25-43, https://doi.org/10.2151/ jmsj1965.44.1_25.

Neelin, J. D., and I. M. Held, 1987: Modeling tropical convergence based on the moist static energy budget. Mon. Wea. Rev., 115, 3-12, https://doi.org/10.1175/1520-0493(1987)115<0003: MTCBOT $>2.0 . C O ; 2$.

, and J.-Y. Yu, 1994: Modes of tropical variability under convective adjustment and the Madden-Julian Oscillation. Part I: Analytical theory. J. Atmos. Sci., 51, 1876-1894, https://doi.org/ 10.1175/1520-0469(1994)051<1876:MOTVUC>2.0.CO;2.

Peters, M. E., and C. S. Bretherton, 2005: A simplified model of the Walker circulation with an interactive ocean mixed layer and 
cloud-radiative feedbacks. J. Climate, 18, 4216-4234, https:// doi.org/10.1175/JCLI3534.1.

$\longrightarrow$, and,- 2006 : Structure of tropical variability from a vertical mode perspective. Theor. Comput. Fluid Dyn., 20, 501524, https://doi.org/10.1007/s00162-006-0034-x.

Raymond, D. J., and Ž. Fuchs, 2007: Convectively coupled gravity and moisture modes in a simple atmospheric model. Tellus, 59A, 627-640, https://doi.org/10.1111/j.1600-0870.2007.00268.x.

- S. L. Sessions, A. H. Sobel, and Ž. Fuchs, 2009: The mechanics of gross moist stability. J. Adv. Model. Earth Syst., 1 (3), https://doi.org/10.3894/JAMES.2009.1.9.

— Ż. Fuchs, S. Gjorgjievska, and S. Sessions, 2015: Balanced dynamics and convection in the tropical troposphere. $J$. $A d v$. Model. Earth Syst., 7, 1093-1116, https://doi.org/10.1002/ 2015 MS000467.

Reed, R. J., and E. E. Recker, 1971: Structure and properties of synoptic-scale wave disturbances in the equatorial western Pacific. J. Atmos. Sci., 28, 1117-1133, https://doi.org/10.1175/ 1520-0469(1971)028<1117:SAPOSS > 2.0.CO;2.

Roundy, P. E., 2012a: Observed structure of convectively coupled waves as a function of equivalent depth: Kelvin waves and the Madden-Julian oscillation. J. Atmos. Sci., 69, 2097-2106, https://doi.org/10.1175/JAS-D-12-03.1.

_ 2012b: The spectrum of convectively coupled Kelvin waves and the Madden-Julian oscillation in regions of low-level easterly and westerly background flow. J. Atmos. Sci., 69, 2107-2111, https://doi.org/10.1175/JAS-D-12-060.1.

Sakaeda, N., and P. E. Roundy, 2016: Gross moist stability and the Madden-Julian Oscillation in reanalysis data. Quart. J. Roy. Meteor. Soc., 142, 2740-2757, https://doi.org/10.1002/qj.2865.

Schumacher, C., R. A. Houze, and I. Kraucunas, 2004: The tropical dynamical response to latent heating estimates derived from the TRMM precipitation radar. J. Atmos. Sci., 61, 1341-1358, https://doi.org/10.1175/1520-0469(2004)061<1341:TTDRTL> 2.0.CO;2.

Seo, K.-H., and W. Wang, 2010: The Madden-Julian oscillation simulated in the NCEP climate forecast system model: The importance of stratiform heating. J. Climate, 23, 4770-4793, https://doi.org/10.1175/2010JCLI2983.1.

Sobel, A. H., 2007: Simple models of ensemble-averaged precipitation and surface wind, given the SST. The Global Circulation of the Atmosphere, T. Schneider and A. H. Sobel, Eds., Princeton University Press, 219-251.

— hot spots. J. Climate, 16, 3978-3992, https://doi.org/10.1175/ 1520-0442(2003)016<3978:ASTMOS>2.0.CO;2.

— , and E. Maloney, 2013: Moisture modes and the eastward propagation of the MJO. J. Atmos. Sci., 70, 187-192, https:// doi.org/10.1175/JAS-D-12-0189.1.

__, J. Nilsson, and L. M. Polvani, 2001: The weak temperature gradient approximation and balanced tropical moisture waves. J. Atmos. Sci., 58, 3650-3665, https://doi.org/10.1175/15200469(2001)058<3650:TWTGAA > 2.0.CO;2.

- S. Wang, and D. Kim, 2014: Moist static energy budget of the MJO during DYNAMO. J. Atmos. Sci., 71, 4276-4291, https:// doi.org/10.1175/JAS-D-14-0052.1.

Straub, K. H., and G. N. Kiladis, 2002: Observations of a convectively coupled Kelvin wave in the eastern Pacific ITCZ. J. Atmos. Sci., 59, 30-53, https://doi.org/10.1175/15200469(2002)059<0030:OOACCK > 2.0.CO;2.

- , and -2003 : The observed structure of convectively coupled Kelvin waves: Comparison with simple models of coupled wave instability. J. Atmos. Sci., 60, 1655-1668, https://doi.org/ 10.1175/1520-0469(2003)060<1655:TOSOCC >2.0.CO;2.

_, P. T. Haertel, and G. N. Kiladis, 2010: An analysis of convectively coupled Kelvin waves in 20 WCRP CMIP3 global coupled climate models. J. Climate, 23, 3031-3056, https:// doi.org/10.1175/2009JCLI3422.1.

Su, H., and J. D. Neelin, 2002: Teleconnection mechanisms for tropical Pacific descent anomalies during El Niño. J. Atmos. Sci., 59, 2694-2712, https://doi.org/10.1175/1520-0469(2002) 059<2694:TMFTPD $>2.0$.CO;2.

Sugiyama, M., 2009: The moisture mode in the quasi-equilibrium tropical circulation model. Part I: Analysis based on the weak temperature gradient approximation. J. Atmos. Sci., 66, 15071523, https://doi.org/10.1175/2008JAS2690.1.

Sumi, Y., and H. Masunaga, 2016: A moist static energy budget analysis of quasi-2-day waves using satellite and reanalysis data. J. Atmos. Sci., 73, 743-759, https://doi.org/10.1175/JASD-15-0098.1.

_ quasi-2-day waves. J. Atmos. Sci., 76, 2005-2022, https:// doi.org/10.1175/JAS-D-19-0092.1.

Takayabu, Y. N., 1994: Large-scale cloud disturbances associated with equatorial waves Part I: Spectral features of the cloud disturbances. J. Meteor. Soc. Japan, 72, 433-449, https:// doi.org/10.2151/jmsj1965.72.3_433.

_ and T. Nitta, 1993: 3-5 day-period disturbances coupled with convection over the tropical Pacific Ocean. J. Meteor. Soc. Japan, 71, 221-246, https://doi.org/10.2151/jmsj1965.71.2_221.

- K. Lau, and C. Sui, 1996: Observation of a quasi-2-day wave during TOGA COARE. Mon. Wea. Rev., 124, 1892-1913, https://doi.org/10.1175/1520-0493(1996)124<1892:OOAQDW> 2.0.CO;2.

Wheeler, M., and G. N. Kiladis, 1999: Convectively coupled equatorial waves: Analysis of clouds and temperature in the wavenumber-frequency domain. J. Atmos. Sci., 56, 374-399, https://doi.org/10.1175/1520-0469(1999)056<0374:CCEWAO > 2.0.CO;2.

Wolding, B. O., and E. D. Maloney, 2015: Objective diagnostics and the Madden-Julian oscillation. Part II: Application to moist static energy and moisture budgets. J. Climate, 28, 77867808, https://doi.org/10.1175/JCLI-D-14-00689.1.

,-- , and M. Branson, 2016: Vertically resolved weak temperature gradient analysis of the Madden-Julian Oscillation in SP-CESM. J. Adv. Model. Earth Syst., 8, 1586-1619, https:// doi.org/10.1002/2016MS000724.

Yasunaga, K., and B. Mapes, 2012a: Differences between more divergent and more rotational types of convectively coupled equatorial waves. Part I: Space-time spectral analyses. J. Atmos. Sci., 69, 3-16, https://doi.org/10.1175/JAS-D-11-033.1.

$\longrightarrow$, and $-2012 \mathrm{~b}$ : Differences between more divergent and more rotational types of convectively coupled equatorial waves. Part II: Composite analysis based on space-time filtering. J. Atmos. Sci., 69, 17-34, https://doi.org/10.1175/JASD-11-034.1.

—, S. Yokoi, K. Inoue, and B. E. Mapes, 2019: Space-time spectral analysis of the moist static energy budget equation. J. Climate, 32, 501-529, https://doi.org/10.1175/JCLI-D-180334.1.

Zhang, C., 2013: Madden-Julian oscillation: Bridging weather and climate. Bull. Amer. Meteor. Soc., 94, 1849-1870, https:// doi.org/10.1175/BAMS-D-12-00026.1. 